\title{
Three-loop soft function for energetic electroweak boson production at hadron colliders
}

\author{
Ze Long Liu ${ }^{a, b}$ and Maximilian Stahlhofen ${ }^{c}$ \\ ${ }^{a}$ PRISMA ${ }^{+}$Cluster of Excellence \& Mainz Institute for Theoretical Physics, \\ Johannes Gutenberg University, \\ 55099 Mainz, Germany \\ ${ }^{b}$ Theoretical Division, Los Alamos National Laboratory, \\ Los Alamos, NM 87545, U.S.A. \\ ${ }^{c}$ Albert-Ludwigs-Universität Freiburg, Physikalisches Institut, \\ D-79104 Freiburg, Germany \\ E-mail: zelongliu@lanl.gov, \\ maximilian.stahlhofen@physik.uni-freiburg.de
}

ABSTRACT: We calculate the three-loop soft function for the production of an electroweak boson (Higgs, $\gamma, W^{ \pm}, Z$ ) with large transverse momentum at a hadron collider. It is the first time a soft function for a three-parton process is computed at next-to-next-to-nextto-leading order $\left(\mathrm{N}^{3} \mathrm{LO}\right)$. As a technical novelty, we perform the calculation in terms of forward-scattering-type loop diagrams rather than evaluating phase space integrals. Our three-loop result contains color-tripole contributions and explicitly confirms predictions on the universal infrared structure of QCD scattering amplitudes with three massless parton legs. The soft function is a central ingredient in the factorized cross section for electroweak boson production near the kinematic endpoint (threshold), where the invariant mass of the recoiling hadronic radiation is small compared to its transverse momentum. Our result is required for predictions of the near-threshold cross sections at $\mathrm{N}^{3} \mathrm{LO}$ and for the resummation of threshold logarithms at primed next-to-next-to-next-to-leading logarithmic $\left(\mathrm{N}^{3} \mathrm{LL}^{\prime}\right)$ accuracy.

KEYwords: Effective Field Theories, Perturbative QCD, Renormalization Group, Resummation

ArXiv EPrint: 2010.05861 


\section{Contents}

1 Introduction 1

2 Threshold factorization and soft function definition 3

$\begin{array}{llr}3 & \text { Dispersive method } & 6\end{array}$

4 Calculation $\quad 10$

5 Results $\quad \mathbf{1 2}$

5.1 Anomalous dimension 14

$\begin{array}{lll}5.2 & \text { Renormalized results } & 16\end{array}$

6 Conclusion $\quad 20$

$\begin{array}{ll}\text { A Three-loop anomalous dimensions } & 21\end{array}$

$\begin{array}{ll}\text { B Bare data } & 24\end{array}$

C Definition of integral families $\quad 26$

$\begin{array}{ll}\text { D Feynman parameter integrations with HyperInt } & 27\end{array}$

\section{Introduction}

The production of an electroweak (EW) boson (Higgs, $\gamma, W^{ \pm}$or $Z$ ) with sizable transverse momentum is among the most fundamental scattering processes at hadron colliders like the LHC. The corresponding cross sections are experimentally and theoretically relatively clean observables and allow precision phenomenology. Their measurements provide excellent tests of the Standard Model, and probe the parton distributions inside the colliding hadrons at small distances. EW gauge boson production at large transverse momenta also represents an important background to Higgs measurements and new physics searches. On the other hand the Higgs transverse momentum spectrum plays e.g. an important role for the analysis of the Higgs couplings.

Moderate and large transverse momenta of EW bosons in hadron collisions are generated primarily by the recoil against hard QCD radiation (jets). Corresponding tree-level processes with one hard final state parton are depicted in figure 1. Compared to the inclusive EW boson production the leading order (LO) contribution therefore contains an additional power of the strong coupling constant $\alpha_{s}$, and it is technically harder to achieve the same level of accuracy on the theory side. In the last couple of years fixedorder QCD predictions for EW boson production with nonzero transverse momentum have 

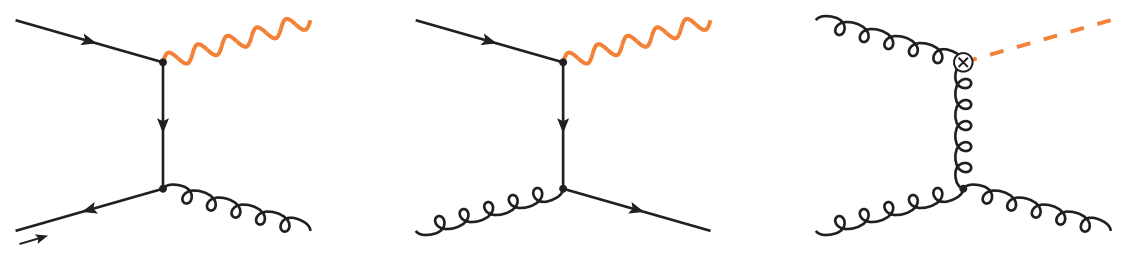

Figure 1. Tree-level Feynman diagrams for electroweak boson production with large transverse momentum at hadron colliders. For EW gauge boson (orange wiggly lines) production example diagrams of two different production channels are shown.

reached NNLO precision [1-16]. Depending on the kinematic region and the type of process the NNLO corrections can be substantial. For example the NNLO QCD corrections to Higgs +1 jet production at the LHC enhance the cross section by roughly $20 \%$, while the uncertainties from scale variations still amount to about $10 \%$ [2]. In view of future precision measurements a further reduction of the theory error would be desirable.

Whereas full QCD N3 $\mathrm{LO}$ results seem currently out of reach, threshold approximations and the corresponding resummation of threshold logarithms can certainly improve the current state-of-the-art fixed-order predictions, at least at large transverse momenta. ${ }^{1}$ In the limit, where the transverse momentum of the EW boson becomes maximal (at fixed rapidity), the invariant mass of the recoiling hadronic radiation vanishes and vice versa. Close to this threshold all final state QCD radiation is either soft or anti-collinear to the direction of the EW boson and the production cross section can be factorized into a convolution of hard, jet, and soft functions $[17,18]$. Within the framework of soft-collinear effective theory (SCET) [19-24] the jet and soft functions are expressed as effective operator matrix elements, while the hard function is a matching coefficient. Each of the factorization functions obeys a corresponding renormalization group equation (RGE). Solving these and evolving the functions from the respective physical hard, jet, and soft scales to a common renormalization scale systematically resums large threshold logarithms (of the ratio between the transverse momentum and the hadronic invariant mass). In this way near-threshold predictions for cross sections with NNLL and partial $\mathrm{N}^{3} \mathrm{LL}$ resummation along with the corresponding NNLO threshold corrections were obtained [18, 25-30].

In order to improve the predictions for EW boson production near threshold to $\mathrm{N}^{3} \mathrm{LO}$ and (resummed) $\mathrm{N}^{3} \mathrm{LL}^{\prime}$ accuracy ${ }^{2}$ the factorization functions are required at three-loop order. Given the recent progress in the calculation of $\mathrm{N}^{3} \mathrm{LO}$ four-particle scattering amplitudes in QCD [32, 33], there is hope that also the three-loop virtual QCD corrections to EW boson + jet production, constituting the hard function, become available in the not-too-far future. The relevant quark and gluon jet functions were computed at three loops in refs. [34, 35]. The present paper is dedicated to the calculation of the three-loop soft function.

The soft function is given by a phase space integral constrained by the threshold measurement over a squared matrix element with one outgoing and two incoming Wilson lines

\footnotetext{
${ }^{1}$ Often the threshold terms amount to the bulk of the corrections at a given order and the cross sections computed in the threshold limit represent indeed good approximations also at moderate transverse momenta.

${ }^{2}$ For details and advantages of the primed counting, see e.g. ref. [31].
} 
along widely separated lightlike directions. Although the relevant two-loop single [36], oneloop double [37], and tree-level triple emission [38] soft currents are available, performing the necessary phase space integrations is a difficult task. We therefore follow a different approach. Generalizing the 'dispersive method' we used to calculate the two-parton soft function for heavy-to-light decays in ref. [39] we express our three-parton soft function in terms of a single Wilson line correlator corresponding to a forward scattering process. The phase space integrations then translate to loop integrations of Feynman diagrams with one internal and four external Wilson lines. This trick allows us to directly apply modern multi-loop technology and makes the three-loop calculation of the soft function relatively straight-forward to carry out.

Our paper is organized as follows. In section 2 we briefly discuss the factorization formula for the near-threshold production of EW bosons at the LHC and define the soft function employing the color space formalism. In section 3 we devise the 'dispersive method' to compute the soft function in terms of loop diagrams. Section 4 describes the three-loop calculation in some detail and we present our results for the soft function in section 5 , including a discussion of its divergence and color structure. We conclude in section 6 .

\section{Threshold factorization and soft function definition}

In order to put the calculation performed in this paper into context we consider EW boson $(Y=\gamma, W, Z, h)$ production at the LHC, i.e. the process $p p \rightarrow Y+X$. The 'hadronic' invariant mass of the final state particles in $X$ (including the proton remnants) is

$$
M_{X}^{2}=\left(P_{1}+P_{2}-p_{Y}\right)^{2},
$$

where $P_{1,2}$ are the proton momenta. For $M_{X} \rightarrow 0$ the particles in $X$ form a collimated jet and any additional radiation must be soft. In this threshold limit the cross section factorizes, which allows the resummation of large logarithms $\sim \log \left(M_{X} / E_{\mathrm{cm}}\right)$ with $E_{\mathrm{cm}}$ being the center of mass energy of the colliding protons [17].

Defining the kinematic invariants at partonic level as

$$
\hat{s}=\left(x_{1} P_{1}+x_{2} P_{2}\right)^{2}=x_{1} x_{2} E_{\mathrm{cm}}^{2}, \quad \hat{t}=\left(x_{1} P_{1}-p_{Y}\right)^{2}-m_{Y}^{2}, \quad \hat{u}=\left(x_{2} P_{2}-p_{Y}\right)^{2}-m_{Y}^{2},
$$

we can express the invariant hadronic mass as

$$
\begin{aligned}
M_{X}^{2}=\left(E_{\mathrm{cm}}^{2}+m_{Y}^{2}\right)\left(1-\frac{m_{\perp}}{m_{\perp}^{\max }}\right) & =\left[\left(1-x_{1}\right) P_{1}+\left(1-x_{2}\right) P_{2}+p_{X}\right]^{2} \\
& =m_{X}^{2}+(\hat{s}+\hat{t})\left(1-x_{1}\right)+(\hat{s}+\hat{u})\left(1-x_{2}\right)+\mathcal{O}\left(M_{X}^{4}\right),
\end{aligned}
$$

where $m_{X}^{2}=p_{X}^{2}$, with $p_{X}=x_{1} P_{1}+x_{2} P_{2}-p_{Y}$, is the total invariant mass of the final state partons (excluding the proton remnants), $x_{1,2}$ are the fractions of the proton momenta carried by the incoming partons of the hard scattering process, $m_{Y}$ is the mass of the EW boson, and $m_{\perp}=\sqrt{p_{T}^{2}+m_{Y}^{2}}$ is its transverse mass. The maximal transverse mass for fixed rapidity $y$ of the EW boson is given by $\left(m_{\perp}^{\max }\right)^{2}=\left(E_{\mathrm{cm}}^{2}+m_{Y}^{2}\right)^{2} /\left(2 E_{\mathrm{cm}} \cosh y\right)^{2}$. 
From eq. (2.3) it is apparent that $M_{X} \rightarrow 0$ requires $m_{X} \rightarrow 0$ and $x_{1,2} \rightarrow 1$ simultaneously. We also see that this limit is approached for large transverse momenta of $Y$.

The corresponding factorized near-threshold cross section as derived in SCET [18] for the channel $a b \rightarrow c Y$ with $\{a, b, c\}=\{q, \bar{q}, g\},\{q, g, q\},\{\bar{q}, g, \bar{q}\}$, or $\{g, g, g\}$ takes the form ${ }^{3}$

$$
\begin{aligned}
\frac{\mathrm{d}^{2} \sigma_{a b}}{\mathrm{~d} p_{T} \mathrm{~d} y} \propto & \frac{\mathrm{d}^{2} \sigma_{a b}}{\mathrm{~d} M_{X}^{2} \mathrm{~d} y} \propto \int \mathrm{d} x_{1} \int \mathrm{d} x_{2} \int \mathrm{d} m^{2} \int \mathrm{d} \omega H_{a b}\left(\hat{s}, \hat{t}, \hat{u}, m_{Y}, \mu\right) f_{a}\left(x_{1}, \mu\right) f_{b}\left(x_{2}, \mu\right) J_{c}\left(m^{2}, \mu\right) \\
& \times S_{a b}\left(n_{i j}, \omega, \mu\right) \delta\left[M_{X}^{2}-m^{2}-2 E_{J} \omega-(\hat{s}+\hat{t})\left(1-x_{1}\right)-(\hat{s}+\hat{u})\left(1-x_{2}\right)\right] . \quad(2.4)
\end{aligned}
$$

The parton distribution functions (PDFs) $f_{i}(x, \mu)$ are evaluated close to their endpoints at $x=1$. The hard function $H_{a b}$ corresponds to a SCET Wilson coefficient obtained from matching SCET currents to full QCD matrix elements at the hard scale $\sim E_{\mathrm{cm}} \sim p_{T} \approx p_{T}^{\max }$ and contains the full QCD virtual corrections to the hard scattering process. It depends on the hard kinematics and is known to two-loop order for $Y=\gamma, W, Z, h$ [40-43]. The jet function $J_{c}$ describes the collinear radiation along the jet direction $n_{J}^{\mu}$, which is anticollinear to the direction of $Y,{ }^{4}$ and only depends on the type of parton initiating the jet and its virtuality $\sim M_{X}$. The respective three-loop results were obtained in refs. [34, 35].

The effects of soft wide-angle radiation (with momenta $\sim M_{X}^{2} / E_{\mathrm{cm}}$ ) are encoded in the soft function $S_{a b}$. It depends on the color representation of the three hard partons and the total momentum component in $\bar{n}_{J}^{\mu}$ direction of the soft partons. We also indicated a dependence on $n_{i j} \equiv n_{i} \cdot n_{j}$ with $i, j=1,2, J$, where the $n_{i}^{\mu}$ denotes the lightlike proton and jet directions in Minkowski space $\left(n_{i}^{2}=0\right)$, respectively. This dependence is however such that it exactly cancels the dependence on the jet energy $E_{J}=\left[(\hat{s}+\hat{t})(\hat{s}+\hat{u}) n_{12} /\left(2 n_{1 J} n_{2 J} \hat{s}\right)\right]^{1 / 2}$ in eq. (2.4) as can be made manifest by rescaling $\omega$ [18]. The soft function is the same for any $Y$ and was calculated at two loops in ref. [44]. In the present paper we compute it at three loops, i.e. $\mathcal{O}\left(\alpha_{s}^{3}\right)$.

For later reference we also quote the Laplace transform of eq. (2.4)

$$
\begin{aligned}
\frac{\mathrm{d}^{2} \tilde{\sigma}_{a b}}{\mathrm{~d} Q^{2} \mathrm{~d} y} & =\int_{0}^{\infty} \mathrm{d} M_{X}^{2} \exp \left(-\frac{M_{X}^{2}}{Q^{2} e^{\gamma_{E}}}\right) \frac{\mathrm{d}^{2} \sigma_{a b}}{\mathrm{~d} M_{X}^{2} \mathrm{~d} y} \\
& \propto H_{a b}\left(\hat{s}, \hat{t}, \hat{u}, m_{Y}, \mu\right) \tilde{f}_{a}\left(\tau_{1}, \mu\right) \tilde{f}_{b}\left(\tau_{2}, \mu\right) \tilde{j}_{c}\left(Q^{2}, \mu\right) \tilde{s}_{a b}(\kappa, \mu),
\end{aligned}
$$

where the convolutions turned into simple products of $\tilde{f}_{i}, \tilde{J}_{c}$, and $\tilde{s}_{a b}$, which denote the PDFs, jet, and soft functions in Laplace space [18], respectively, and

$$
\tau_{1}=\frac{Q^{2}}{\hat{s}+\hat{t}}, \quad \tau_{2}=\frac{Q^{2}}{\hat{s}+\hat{u}}, \quad \kappa=\frac{Q^{2}}{2 E_{J}} .
$$

In section 5.1, we derive the three-loop anomalous dimension of the soft function in Laplace space via the renormalization group (RG) invariance of eq. (2.5) and check the consistency with our explicit calculation.

\footnotetext{
${ }^{3}$ The factorization was performed here on the partonic level for small $m_{X}$. The factorized partonic cross section is then convoluted with the (threshold) PDFs to obtain eq. (2.4).

${ }^{4}$ We define $n_{i}^{2}=0$ and $\bar{n}_{i} \cdot n_{i}=2$ for $i=1,2, J$.
} 
In SCET the PDFs, the jet function, and the soft function are defined as operator matrix elements. For illustration we quote here the relevant (unrenormalized) expressions for the quark and anti-quark PDFs of a proton $\left(p_{n_{i}}\right)$ with momentum $P^{-} n_{i}^{\mu} / 2$ employing the SCET label formalism [23, 45]:

$$
\begin{aligned}
& f_{q}(x)=\left\langle p_{n_{i}}\left(P^{-}\right)\left|\theta(x) \bar{\chi}_{n_{i}}(0) \frac{\not_{i}}{2}\left[\delta\left(x P^{-}-\overline{\mathcal{P}}_{n_{i}}\right) \chi_{n_{i}}(0)\right]\right| p_{n_{i}}\left(P^{-}\right)\right\rangle, \\
& f_{\bar{q}}(x)=\left\langle p_{n_{i}}\left(P^{-}\right)\left|\theta(x) \operatorname{tr}\left\{\frac{\hbar_{i}}{2} \chi_{n_{i}}(0)\left[\delta\left(x P^{-}-\overline{\mathcal{P}}_{n_{i}}\right) \bar{\chi}_{n_{i}}(0)\right]\right\}\right| p_{n_{i}}\left(P^{-}\right)\right\rangle .
\end{aligned}
$$

The composite quark field operator $\chi_{n_{i}}$ is gauge-invariant w.r.t. collinear gauge transformations and includes a collinear Wilson line in its definition, see e.g. ref. [45] for details. The label momentum operator $\overline{\mathcal{P}}_{n_{i}}$ returns the total large light-cone (minus) momentum component of the $n_{i}$-collinear fields it acts on. A similar expression holds for the gluon PDF $[23,45]$. The jet function and PDFs have trivial color structure, i.e. they do not have open color indices.

In contrast, the hard and soft functions can be regarded as color tensors with one index for each parton in the hard scattering amplitude and one for each parton in the complex conjugate amplitude. Corresponding color indices of soft and hard functions are pairwise contracted in the factorized cross section. (In eqs. (2.4) and (2.5) color indices have been suppressed for the sake of compactness.) A convenient way to deal with the colors structure of scattering processes with more than two partons is the color-space formalism [46, 47]. Applying it to our factorized cross section the soft function represents a square matrix in color space acting to the left and right on color vectors corresponding to the hard scattering amplitude and its complex conjugate. In general we write for the hard function and the corresponding hard amplitude ${ }^{5}$

$$
H_{a_{1} a_{2} \ldots b_{1} b_{2} \ldots} \propto C_{a_{1} a_{2} \ldots}\left(C_{b_{1} b_{2} \ldots}\right)^{*}, \quad C_{a_{1} a_{2} \ldots}=\sum_{k}\left\langle\left. a_{1} a_{2} \ldots\right|_{k} C_{k}\right.
$$

where the $a_{i}$ (and $b_{i}$ ) are color indices of the parton legs and $\left\langle\left. a_{1} a_{2} \ldots\right|_{k}\right.$ are (not necessarily orthonormal) vectors spanning the color space. In the special case of three hard partons the color space is only one-dimensional and we define ${ }^{6}$

$$
\left\langle a_{1} a_{2} a_{J}|=| a_{1} a_{2} a_{J}\right\rangle^{*}= \begin{cases}t_{a_{2} a_{1}}^{a_{J}} & \text { for the } q \bar{q} \rightarrow g \text { channel, } \\ t_{a_{J} a_{1}}^{a_{2}} & \text { for the } q g \rightarrow q \text { channel, } \\ \text { if }{ }^{a_{2} a_{J} a_{1}} & \text { for the } g g \rightarrow g \text { channel, }\end{cases}
$$

where $t_{b c}^{a}$ and if ${ }^{b a c}$ are the $\mathrm{SU}\left(N_{c}\right)$ generators in the fundamental and the adjoint representation, respectively. ${ }^{7}$ We thus have (for each production channel)

$$
H_{a_{1} a_{2} a_{J} b_{1} b_{2} b_{J}} S_{a_{1} a_{2} a_{J} b_{1} b_{2} b_{J}}=H\left\langle a_{1} a_{2} \ldots|\boldsymbol{S}| a_{1} a_{2} a_{J}\right\rangle=H S\left\langle a_{1} a_{2} a_{J} \mid a_{1} a_{2} a_{J}\right\rangle,
$$

\footnotetext{
${ }^{5}$ The hard amplitude $C$ equals the QCD scattering amplitude with hard parton legs, where in the course of the SCET matching procedure, the IR divergences are subtracted.

${ }^{6}$ Here and in the following we often neglect the $\bar{q} g \rightarrow \bar{q}$ channel, because it is trivially related to the $q g \rightarrow q$ channel by charge conjugation. In QCD therefore all physical results for the two channels are the same.

${ }^{7}$ The totally-symmetric tensor $d^{a b c}=\operatorname{tr}\left[\left\{t^{a}, t^{b}\right\} t^{c}\right] / T_{F}$ is ruled out by charge conjugation symmetry of QCD as another possible color structure for the $g g \rightarrow g$ amplitude, see e.g. ref. [48].
} 
where $S_{a_{1} a_{2} \ldots}$ represents the soft function as a tensor with color indices and $\boldsymbol{S}$ represents the soft function as an operator acting on color space vectors, which is proportional to the unit operator $(\boldsymbol{S}=S \mathbb{1})$ in our one-dimensional color space.

Using this notation the soft function operator is defined by [18]

$$
\boldsymbol{S}(\omega)=\left\langle 0\left|\overline{\mathrm{T}}\left[Y_{J, \text { out }}^{\dagger}(0) Y_{1, \text { in }}^{\dagger}(0) Y_{2, \text { in }}^{\dagger}(0)\right] \delta\left(\omega-n_{J} \cdot \hat{p}\right) \mathrm{T}\left[Y_{J, \text { out }}(0) Y_{1, \text { in }}(0) Y_{2, \text { in }}(0)\right]\right| 0\right\rangle,
$$

where $\mathrm{T}(\overline{\mathrm{T}})$ indicates (anti-)time ordering, $\hat{p}^{\mu}$ is the soft momentum operator, and

$$
\begin{gathered}
Y_{i, \text { in }}(x)=\overline{\mathrm{P}} \exp \left[-\mathrm{i} g \int_{-\infty}^{0} \mathrm{~d} s n_{i} \cdot A^{c}\left(x+s n_{i}\right) \boldsymbol{T}_{i}^{c}\right], \\
Y_{i, \text { out }}(x)=\mathrm{P} \exp \left[+\mathrm{i} g \int_{0}^{\infty} \mathrm{d} s n_{i} \cdot A^{c}\left(x+s n_{i}\right) \boldsymbol{T}_{i}^{c}\right],
\end{gathered}
$$

are the soft Wilson lines for an incoming and outgoing parton $i$, respectively. The symbol $\mathrm{P}(\overline{\mathrm{P}})$ denotes (anti-)path ordering of the color charge operators $\boldsymbol{T}_{i}^{c}$ and the associated (ultra)soft SCET gluon field operators $A_{\mu}^{c}(x)$. The action of the $\boldsymbol{T}_{i}^{c}$ on color space vectors is defined by

$$
\left\langle a_{1} \ldots a_{i} \ldots a_{n}\left|\boldsymbol{T}_{i}^{c}\right| a_{1} \ldots a_{i} \ldots a_{n}\right\rangle \equiv\left\langle a_{1} \ldots b_{i} \ldots a_{n}\left|T_{a_{i} b_{i}}^{c}\right| a_{1} \ldots a_{i} \ldots a_{n}\right\rangle
$$

with

$$
T_{a_{i} b_{i}}^{c} \equiv \begin{cases}t_{a_{i} b_{i}}^{c} & \text { if parton } i \text { is a outgoing quark or incoming antiquark } \\ -t_{b_{i} a_{i}}^{c} & \text { if parton } i \text { is an incoming quark or outgoing antiquark } \\ \mathrm{i} f^{a_{i} c b_{i}} & \text { if parton } i \text { is a gluon. }\end{cases}
$$

Note that $\boldsymbol{T}_{i}^{c} \boldsymbol{T}_{i}^{c}=C_{R} \mathbb{1}$, where $C_{R}$ is the quadratic Casimir of the color representation $R$ of parton $i$ ( $R=F$ for (anti)quarks and $R=A$ for gluons) and

$$
\sum_{i=1}^{n}\left\langle a_{1} \ldots a_{i} \ldots a_{n}\left|\boldsymbol{T}_{i}^{c}=\sum_{i=1}^{n} \boldsymbol{T}_{i}^{c}\right| a_{1} \ldots a_{i} \ldots a_{n}\right\rangle=0
$$

due to color conservation in the scattering amplitudes. Color charge operators of different partons commute trivially, i.e. $\left[\boldsymbol{T}_{i}^{a}, \boldsymbol{T}_{j}^{b}\right]=0$ for $i \neq j$, while $\left[\boldsymbol{T}_{i}^{a}, \boldsymbol{T}_{i}^{b}\right]=\mathrm{i} f^{a b c} \boldsymbol{T}_{i}^{c}$.

\section{Dispersive method}

The soft function defined in eq. (2.12) represents an integral of a squared matrix element of one outgoing and two incoming Wilson lines. This can be made explicit by inserting a complete set of (soft final) states $(X)$,

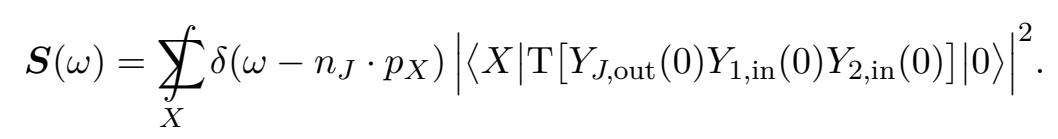

Rather than evaluating phase space integrals we would like to compute the soft function in terms of forward-scattering-type loop diagrams. A similar 'dispersive' method was used for 
the calculation of the soft function for heavy-to-light decays near the kinematic endpoint as detailed in ref. [39].

Following this approach, we will rewrite the $\delta$-function in eq. (2.12) as a (momentumspace Wilson line) propagator-type expression using

$$
\delta(x)=\mp \frac{1}{\pi} \operatorname{Im}\left[\frac{1}{x \pm \mathrm{i} \delta}\right]= \pm \frac{1}{\pi} \operatorname{Re}\left[\frac{\mathrm{i}}{x \pm \mathrm{i} \delta}\right],
$$

where i $\delta$ represents an infinitesimally small positive imaginary part. Our goal is to express the soft function in terms of a single time-ordered (forward-scattering) matrix element, which can be evaluated using ordinary (Wilson line) momentum space Feynman rules. As usual in momentum space perturbation theory the time-ordering is effectively implemented via the causal 'i0' prescriptions for the propagators in the corresponding Feynman graphs. Using eq. (3.2) means that we will introduce another independent imaginary infinitesimal in the computation and special care has to be taken in order to avoid unphysical imaginary parts due to interference of i $\delta$ and i0 in loop graphs giving rise to physical thresholds. ${ }^{8}$

Concretely, we implement the $\delta$-function in eq. (2.12) as follows

$$
\begin{aligned}
\boldsymbol{S}(\omega)=\left\langle O^{\dagger} \delta\left(\omega-n_{J} \cdot \hat{p}\right) O\right\rangle & =\frac{1}{2 \pi}\left(\left\langle O^{\dagger} \operatorname{Re}\left[\frac{\mathrm{i}}{\omega-n_{J} \cdot \hat{p}+\mathrm{i} \delta}\right] O\right\rangle-\left\langle O^{\dagger} \operatorname{Re}\left[\frac{\mathrm{i}}{\omega-n_{J} \cdot \hat{p}-\mathrm{i} \delta}\right] O\right\rangle\right) \\
& =\frac{1}{2 \pi} \operatorname{Re}\left[\left\langle O^{\dagger} \frac{\mathrm{i}}{\omega-n_{J} \cdot \hat{p}+\mathrm{i} \delta} O\right\rangle-\left\langle O^{\dagger} \frac{\mathrm{i}}{\omega-n_{J} \cdot \hat{p}-\mathrm{i} \delta} O\right\rangle\right], \\
& =\frac{1}{2 \pi} \operatorname{Re}\left[\operatorname{Disc}_{\omega}\left\langle O^{\dagger} \frac{\mathrm{i}}{\omega-n_{J} \cdot \hat{p}} O\right\rangle\right],
\end{aligned}
$$

where $O \equiv \mathrm{T}\left[Y_{J, \text { out }}(0) Y_{1, \text { in }}(0) Y_{2, \text { in }}(0)\right],\langle\ldots\rangle \equiv\langle 0|\ldots| 0\rangle$, and $\operatorname{Disc}_{x} g(x) \equiv \lim _{\beta \rightarrow 0}[g(x+$ $\mathrm{i} \beta)-g(x-\mathrm{i} \beta)]$. At first sight taking the real part in eq. (3.3) may seem redundant. It will however become important due to our treatment of i $\delta$ and i0 in the following derivation. We will illustrate the necessity of taking the real part in our dispersive approach for the soft function explicitly at two loops at the end of this section.

Note that the operator matrix element in the last line of eq. (3.3) alone is ambiguous. We therefore conveniently define

$$
\sigma(\omega) \equiv\left\langle 0\left|\overline{\mathrm{T}}\left[Y_{J, \text { out }}^{\dagger}(0) Y_{1, \text { in }}^{\dagger}(0) Y_{2, \text { in }}^{\dagger}(0)\right] \frac{\mathrm{i}}{\omega-n_{J} \cdot \hat{p}+\mathrm{i} 0} \mathrm{~T}\left[Y_{J, \text { out }}(0) Y_{1, \text { in }}(0) Y_{2, \text { in }}(0)\right]\right| 0\right\rangle .
$$

Here we inserted $+\mathrm{i} 0$ in the denominator in order to obtain a well-defined analytical expression for real $\omega$ and to make $\sigma(\omega)$ directly calculable via Feynman diagrams as we will demonstrate below. With eq. (3.4) we can write

$$
\boldsymbol{S}(\omega)=S(\omega) \mathbb{1}=\frac{1}{2 \pi} \operatorname{Re}\left[\operatorname{Disc}_{\omega} \sigma(\omega)\right]
$$

\footnotetext{
${ }^{8}$ By 'physical threshold' (not to be confused with the large transverse momentum 'threshold' of the EW boson) we refer to a branch cut along physical values of some kinematic invariant the soft function depends on besides $\omega$. The corresponding amplitude for the heavy-to-light soft function discussed in ref. [39] is free of such thresholds. The subtleties related to the difference of i $\delta$ and $\mathrm{i} 0$ are therefore absent in that case and eq. (3.2) can directly be applied with $\mathrm{i} \delta \simeq \mathrm{i} 0$.
} 
where the i $0 \rightarrow 0$ limit inside $\sigma(\omega)$ is understood to be taken before the discontinuity. Note that we could just as well define $\sigma(\omega)$ with i0 $\rightarrow-$ i0, which would lead to $\operatorname{Disc}_{\omega} \sigma(\omega) \rightarrow$ $\left[\operatorname{Disc}_{\omega} \sigma(\omega)\right]^{*}$. Taking the real part in eq. (3.5) removes this ambiguity and is therefore necessary to ensure the consistency with eq. (3.3).

Let us now consider the evaluation of $\sigma(\omega)$. First, we notice that

$$
\sigma(\omega)=\left\langle 0\left|\overline{\mathrm{T}}\left[Y_{1, \text { in }}^{\dagger}(0) Y_{2, \text { in }}^{\dagger}(0)\right] Y_{J, \text { out }}^{\dagger}(0) \frac{\mathrm{i}}{\omega-n_{J} \cdot \hat{p}+\mathrm{i} 0} Y_{J, \text { out }}(0) \mathrm{T}\left[Y_{1, \text { in }}(0) Y_{2, \text { in }}(0)\right]\right| 0\right\rangle,
$$

because the gluon field operators $A_{\mu}^{a}$ inside $Y_{J, \text { out }}^{(\dagger)}$ are already (anti)time-ordered by default as a consequence of the (anti)path-ordering in eq. (2.14), and because the fields at positive times from $Y_{J, \text { out }}^{(\dagger)}$ are already to the left (right) of the fields at negative times in the $\mathrm{T}(\overline{\mathrm{T}})$ product. Using

$$
\frac{\mathrm{i}}{\omega-n_{J} \cdot \hat{p}+\mathrm{i} 0}=\int_{-\infty}^{\infty} \mathrm{d} r \mathrm{e}^{\mathrm{i}\left(\omega-n_{J} \cdot \hat{p}\right) r} \theta(r)=\int_{0}^{\infty} \mathrm{d} r \mathrm{e}^{\mathrm{i}\left(\omega-n_{J} \cdot \hat{p}\right) r},
$$

we can thus write

$$
\begin{aligned}
& \sigma(\omega)=\int_{0}^{\infty} \mathrm{d} r \mathrm{e}^{\mathrm{i} \omega r}\left\langle 0\left|\overline{\mathrm{T}}\left[Y_{1, \text { in }}^{\dagger}(0) Y_{2, \text { in }}^{\dagger}(0)\right] Y_{J, \text { out }}^{\dagger}(0) \mathrm{e}^{-\mathrm{i} n_{J} \cdot \hat{p} r} Y_{J, \text { out }}(0) \mathrm{T}\left[Y_{1, \text { in }}(0) Y_{2, \text { in }}(0)\right]\right| 0\right\rangle \\
& =\int_{0}^{\infty} \mathrm{d} r \mathrm{e}^{\mathrm{i} \omega r}\left\langle 0\left|\overline{\mathrm{T}}\left[Y_{1, \text { in }}^{\dagger}\left(r n_{J}\right) Y_{2, \text { in }}^{\dagger}\left(r n_{J}\right)\right] Y_{J, \text { out }}^{\dagger}\left(r n_{J}\right) Y_{J, \text { out }}(0) \mathrm{T}\left[Y_{1, \text { in }}(0) Y_{2, \text { in }}(0)\right]\right| 0\right\rangle \\
& =\int_{0}^{\infty} \mathrm{d} r \mathrm{e}^{\mathrm{i} \omega r}\left\langle 0\left|\overline{\mathrm{T}}\left[Y_{1, \text { in }}^{\dagger}\left(r n_{J}\right) Y_{2, \text { in }}^{\dagger}\left(r n_{J}\right)\right] \mathrm{P} \exp \left[\mathrm{i} g \int_{0}^{r} \mathrm{~d} s n_{J} \cdot A^{c}\left(s n_{J}\right) \boldsymbol{T}_{J}^{c}\right] \mathrm{T}\left[Y_{1, \text { in }}(0) Y_{2, \text { in }}(0)\right]\right| 0\right\rangle .
\end{aligned}
$$

For an evaluation of $\sigma(\omega)$ via forward scattering Feynman diagrams all field operators in eq. (3.8) must be time-ordered. In the present form they are already time-ordered except for the operators in the $\overline{\mathrm{T}}$ product. We can bring them into time order by the procedure sketched in the following, see also ref. [39] for a similar argument.

We first recombine $\sigma(\omega)$ with the $n_{1}$ - and $n_{2}$-collinear matrix elements, i.e. the PDFs. We can now undo the BPS field redefinition [22] of the collinear fields, which made the decoupling of soft and collinear sectors at leading order in the SCET expansion manifest. While the following arguments hold in general, let us consider the $q \bar{q} \rightarrow g$ channel for concreteness. The resulting soft-collinear matrix element then has the schematic form

$$
\left\langle p_{n_{1}} p_{n_{2}}\left|\bar{\chi}_{n_{1}}\left(r n_{J}\right) \chi_{n_{2}}\left(r n_{J}\right) \mathrm{P} \exp \left[\mathrm{i} g \int_{0}^{r} \mathrm{~d} s n_{J} \cdot A^{c}\left(s n_{J}\right) \boldsymbol{T}_{J}^{c}\right] \chi_{n_{1}}(0) \bar{\chi}_{n_{2}}(0)\right| p_{n_{1}} p_{n_{2}}\right\rangle
$$

where we suppressed the Dirac structure as well as the delta functions fixing the label momentum of the collinear $(\chi)$ fields from eqs. (2.7) and (2.8) for brevity. As $r>0$ the fields in eq. (3.9) are in fact time-ordered. We can make this manifest by inserting the time-ordering operator $\mathrm{T}$ and redo the BPS field redefinitions $\chi_{n_{1}} \rightarrow Y_{1 \text {,in }} \chi_{n_{1}}$ and $\bar{\chi}_{n_{2}} \rightarrow$ $Y_{2, \text { in }} \bar{\chi}_{n_{2}}{ }^{9}$ Note, however, that the consistent interpretation of eq. (3.9) as forward matrix element now requires that $\left\langle p_{n_{1}} p_{n_{2}}\right|={ }_{\text {out }}\left\langle p_{n_{1}} p_{n_{2}}\right|$. This entails that the field redefinition

\footnotetext{
${ }^{9}$ To properly keep track of the color indices, see e.g. the detailed derivation of the factorization theorem based on the BPS field redefinition in ref. [18].
} 


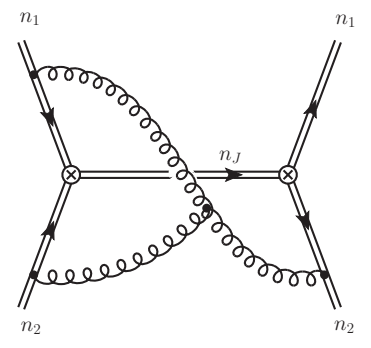

Figure 2. Two-loop diagram contributing to $\sigma(\omega)$ and responsible for the imaginary part of $\operatorname{Disc}_{\omega} \sigma(\omega)$ at this order. The external double lines on the left (right) correspond to the semi-infinite lightlike Wilson lines $Y_{i, \text { in (out) }}^{(\dagger)}$, the middle double line corresponds to the finite-length Wilson line in eq. (3.11). The little arrows indicate the parton flow for a generic parton channel with color charge operators $\boldsymbol{T}_{i}^{c}$ for each Wilson line vertex. The crossed vertices on the left (right) indicate the (complex conjugated) hard amplitude.

of the interpolating (anti)quark fields at time $+\infty$ generating part of the outgoing protonproton state gives rise to an additional factor $Y_{1 \infty} Y_{2 \infty}[49]$ with

$$
Y_{i \infty}=\overline{\mathrm{P}} \exp \left[-\mathrm{i} g \int_{-\infty}^{+\infty} \mathrm{d} s n_{i} \cdot A^{c}\left(x+s n_{i}\right) \boldsymbol{T}_{i}^{c}\right]=Y_{i, \text { in }}\left(r n_{J}\right) Y_{i, \text { out }}^{\dagger}\left(r n_{J}\right)
$$

to the right of the $Y_{i \text {,in }}^{\dagger}$. We emphasize that the color charge operator $\boldsymbol{T}_{i}$ in $Y_{i \text {,out }}$ is still that of an incoming parton $i=1,2$ w.r.t. eq. (2.16).

We can now factorize out the PDFs again, which are matrix elements of local operator products in SCET and as such unaffected by the time-ordering, and finally end up with

$$
\sigma(\omega)=\int_{0}^{\infty} \mathrm{d} r \mathrm{e}^{\mathrm{i} \omega r}\left\langle 0\left|\mathrm{~T}\left[Y_{1, \text { out }}^{\dagger}\left(r n_{J}\right) Y_{2, \text { out }}^{\dagger}\left(r n_{J}\right) \mathrm{P} \exp \left[\mathrm{i} g \int_{0}^{r} \mathrm{~d} s n_{J} \cdot A^{c}\left(s n_{J}\right) \boldsymbol{T}_{J}^{c}\right] Y_{1, \text { in }}(0) Y_{2, \text { in }}(0)\right]\right| 0\right\rangle .
$$

This expression for $\sigma(\omega)$ admits a straightforward evaluation in terms of forward-scatteringtype loop diagrams using QCD Wilson-line Feynman rules in momentum space.

One of the relevant Feynman graphs at two-loop order is shown in figure 2. Applying the Feynman rules and dimensional regularization $(d=4-2 \epsilon)$ we have

$$
\begin{aligned}
& \left.\sigma(\omega)\right|_{\text {Figure } 2}=-16 \pi^{2} \alpha_{s}^{2} n_{12} f^{a b c} \boldsymbol{T}_{1}^{a} \boldsymbol{T}_{2}^{c} \boldsymbol{T}_{2}^{b} \int \frac{\mathrm{d}^{d} l_{1}}{(2 \pi)^{d}} \frac{\mathrm{d}^{d} l_{2}}{(2 \pi)^{d}} \\
& \times \frac{n_{2} \cdot\left(l_{1}+l_{2}\right)}{\left[n_{1} \cdot\left(l_{1}-l_{2}\right)+\mathrm{i} 0\right]\left(n_{2} \cdot l_{2}+\mathrm{i} 0\right)\left(n_{J} \cdot l_{1}+\omega+\mathrm{i} 0\right)\left(n_{2} \cdot l_{1}+\mathrm{i} 0\right)\left(l_{1}^{2}+\mathrm{i} 0\right)\left(l_{2}^{2}+\mathrm{i} 0\right)\left[\left(l_{1}-l_{2}\right)^{2}+\mathrm{i} 0\right]} \\
& =-2 \mathrm{i} C_{S} C_{A} \mathbb{1}\left(\frac{\alpha_{s}}{4 \pi}\right)^{2}(2 \pi)^{2 \epsilon}(-\omega-\mathrm{i} 0)^{-1-4 \epsilon}\left(n_{1 J} n_{2 J}\right)^{2 \epsilon}\left[\left(n_{12}\right)^{-2 \epsilon} \Gamma(1-2 \epsilon) \Gamma^{2}(-\epsilon) \Gamma(2 \epsilon) \Gamma(4 \epsilon)\right. \\
& \left.+\left(-n_{12}-\mathrm{i} 0\right)^{-2 \epsilon} \frac{\Gamma^{2}(-2 \epsilon) \Gamma^{2}(-\epsilon) \Gamma(2 \epsilon) \Gamma(1+4 \epsilon) \Gamma(1+3 \epsilon)}{\Gamma(\epsilon)}\right] \cdot
\end{aligned}
$$

The left-right and up-down mirror graphs yield the exact same result. The color factor $C_{S}$ depends on the channel and is given in eq. (5.3). To obtain the corresponding contribution to the soft function according to eq. (3.5) we first take the discontinuity in $\omega$ (after 


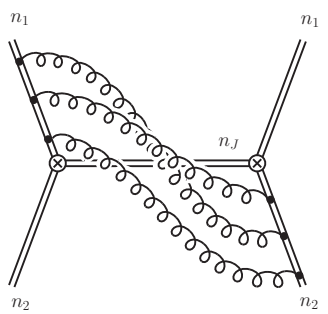

(a)

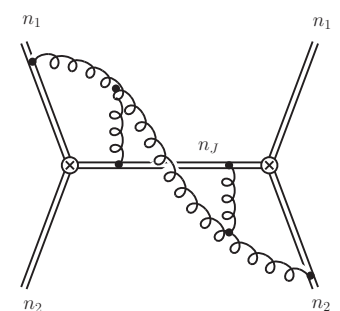

(b)

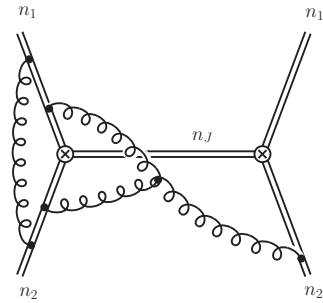

(c)

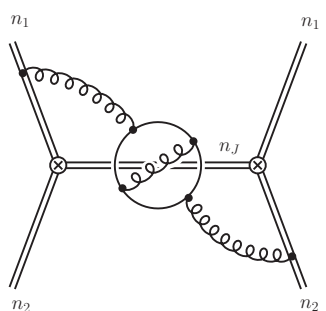

(d)

Figure 3. Examples for three-loop Feynman diagrams that contribute to the soft function $S(\omega)$.

$\omega+\mathrm{i} 0 \rightarrow \omega)$ using

$$
\operatorname{Disc}_{\omega}(-\omega)^{-1-a \epsilon}=-2 \mathrm{i} \sin (\pi a \epsilon) \omega^{-1-a \epsilon} \theta(\omega) .
$$

The resulting expression has an imaginary part from the branch cut in the $\left(-n_{12}-\mathrm{i} 0\right)^{-2 \epsilon}$ factor for (physical) $n_{12}>0$ (related to a physical threshold in the full QCD one-gluon emission amplitude for the process $a b \rightarrow c Y$ ). In a calculation based on eq. (3.1) as performed e.g. in ref. [44] such imaginary parts also appear in the soft amplitude, but cancel in the product with the complex conjugated amplitude. Equivalently, using two-loop diagrams with a unitarity cut (through a single gluon line) the imaginary part cancels between the diagram corresponding to figure 2 and its left-right mirror diagram. In our approach the imaginary part is removed by explicitly by taking the real part in eq. (3.5). At two loops the diagram in figure 2 (together with its mirror graphs) is the only one with an imaginary part after taking the discontinuity. At three loops we found quite a number of such diagrams.

\section{Calculation}

In this section, we present details of our three-loop calculation of the soft function based on eqs. (3.5) and (3.11). The calculation is performed in general covariant gauge with gauge parameter $\xi$, where $\xi=0$ corresponds to Feynman gauge. Ultraviolet (UV) and infrared (IR) divergences are regulated using dimensional regularization $(d=4-2 \epsilon)$.

We generate the relevant three-loop Feynman diagrams with qgraf [50]. Some examples of non-trivial three-loop graphs are shown in figure 3. Based on dimensional counting and investigating the behavior of the corresponding loop integrals under rescaling

$$
n_{1} \rightarrow \lambda_{1} n_{1}, \quad n_{2} \rightarrow \lambda_{2} n_{2}, \quad n_{J} \rightarrow \lambda_{J} n_{J}, \quad \omega \rightarrow \lambda_{J} \omega
$$

we can directly identify already at this stage classes of diagrams that vanish as scaleless integrals, as illustrated in figure 4. We are then left with 1290 non-trivial diagrams to be computed.

These diagrams are further processed by a private Mathematica code [51] which first assigns the momentum space Feynman rules and performs simplifications of the Dirac, Lorentz and color structure. After that the diagrams are expressed as linear combinations of scalar Feynman integrals. Exploiting the $n_{1} \leftrightarrow n_{2}$ interchange symmetry these can be 

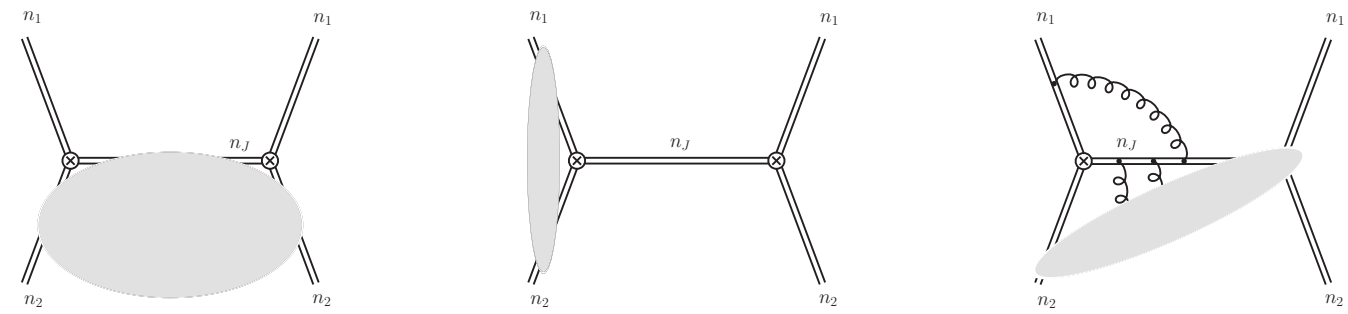

Figure 4. Using the same arguments that lead to eq. (4.4) one can show that (sub)diagrams whose integrand is independent of at least one of the lightlike vectors $n_{i}$ and any external invariant $l_{j}^{2}$ (where $l_{j}$ is an external loop momentum w.r.t. the subloop) vanish in dimensional regularization. Examples for such diagrams are shown in this figure. The gray blob stands for all possible subdiagrams that involve the Wilson lines it overlaps.

mapped onto twenty integral families corresponding to different sets of fifteen linearly independent linear (Wilson line) and quadratic propagators. The mapping of scalar integrals to specific families requires partial-fraction decomposition of linear propagators followed by suitable shifts of the loop momenta. In order to automate the extensive partial fractioning we employed the algorithm described in ref. [52].

Next, we use the public program FIRE5 [53] to perform the integration-by-parts (IBP) reduction of the integrals in each of the twenty integral families to a set of master integrals (MIs). In three of the families we find an additional partial-fraction identity among the MIs returned by FIRE5, reducing their number by one, respectively. We then identify redundant MIs across the families, i.e. subsets of MIs with the same integrand up to loop momentum shifts despite being members of different families. Finally, the sum of all three-loop diagrams contributing to $\sigma(\omega)$ can be expressed as a linear combination (with real $d$-dependent coefficients) of 73 linearly independent MIs belonging to eleven different integral families. In this expression the gauge parameter $\xi$ manifestly cancels out, which represents a strong check of our setup.

The integrals in each family can be written as

$$
G(\vec{a}, \vec{b}, \vec{c}, \vec{e}, \epsilon)=\left(\mathrm{i} \pi^{\frac{d}{2}}\right)^{-3} \int \frac{\mathrm{d}^{d} k_{1} \mathrm{~d}^{d} k_{2} \mathrm{~d}^{d} k_{3}}{\mathcal{D}_{1}^{a_{1}} \mathcal{D}_{2}^{a_{2}} \mathcal{D}_{3}^{a_{3}} \mathcal{D}_{4}^{a_{4}} \mathcal{D}_{5}^{a_{5}} \mathcal{D}_{6}^{a_{6}} \mathcal{D}_{7}^{b_{1}} \mathcal{D}_{8}^{b_{2}} \mathcal{D}_{9}^{b_{3}} \mathcal{D}_{10}^{c_{1}} \mathcal{D}_{11}^{c_{2}} \mathcal{D}_{12}^{c_{3}} \mathcal{D}_{13}^{e_{1}} \mathcal{D}_{14}^{e_{2}} \mathcal{D}_{15}^{e_{3}}},
$$

where the denominators $\mathcal{D}_{1, \cdots, 6}$ correspond to quadratic propagators, and $\mathcal{D}_{7,8,9}, \mathcal{D}_{10,11,12}$, and $\mathcal{D}_{13,14,15}$ correspond to propagators of Wilson lines along the $n_{1}, n_{2}$ and $n_{J}$ directions, respectively. To give an example, one of our integral families is defined by

$$
\begin{array}{rlrl}
\mathcal{D}_{1} & =-k_{1}^{2}, & \mathcal{D}_{2}=-k_{2}^{2}, & \mathcal{D}_{3}=-k_{3}^{2}, \\
\mathcal{D}_{4}=-\left(k_{1}-k_{2}\right)^{2}, & \mathcal{D}_{5}=-\left(k_{2}-k_{3}\right)^{2}, & \mathcal{D}_{6}=-\left(k_{3}-k_{1}\right)^{2}, \\
\mathcal{D}_{7}=-n_{1} \cdot k_{1}, & \mathcal{D}_{8}=-n_{1} \cdot k_{2}, & \mathcal{D}_{9}=-n_{1} \cdot k_{3}, \\
\mathcal{D}_{10}=-n_{2} \cdot k_{1}, & \mathcal{D}_{11}=-n_{2} \cdot k_{2}, & \mathcal{D}_{12}=-n_{2} \cdot k_{3}, \\
\mathcal{D}_{13}=-n_{J} \cdot k_{1}-\omega, & \mathcal{D}_{14}=-n_{J} \cdot k_{2}-\omega, & \mathcal{D}_{15}=-n_{J} \cdot k_{3}-\omega,
\end{array}
$$

where $\mathcal{D}_{i} \rightarrow \mathcal{D}_{i}-\mathrm{i} 0$ in eq. (4.2) is understood. Our definitions of propagator denominators $\mathcal{D}_{i}$ for the remaining ten integral families are given in appendix C. For all eleven families, 
the result of the generic integral in eq. (4.2) is constrained by the scaling properties of its integrand w.r.t. eq. (4.1) to be of the form ${ }^{10}$

$$
\begin{aligned}
G(\vec{a}, \vec{b}, \vec{c}, \vec{e}, \epsilon)= & (-\omega-\mathrm{i} 0)^{3 d-2 A-B-C-E}\left(\frac{n_{12}}{2}\right)^{\frac{3 d}{2}-A-B-C}\left(\frac{n_{1 J}}{2}\right)^{A+C-\frac{3 d}{2}}\left(\frac{n_{2 J}}{2}\right)^{A+B-\frac{3 d}{2}} \\
& \times I(\vec{a}, \vec{b}, \vec{c}, \vec{e}, \epsilon),
\end{aligned}
$$

where $A=\sum_{i} a_{i}, B=\sum_{i} b_{i}, C=\sum_{i} c_{i}$ and $E=\sum_{i} e_{i}$. The dependence on the external kinematics is thus completely fixed and factored out. What is left to be computed is the dimensionless, in general complex, function $I(\vec{a}, \vec{b}, \vec{c}, \vec{e}, \epsilon)$. In fact, because of eq. (3.5), we can safely set $\omega=-1$ and only evaluate the real parts of the relevant MIs as an expansion in $\epsilon$. The $\omega$ dependence of the MIs can finally be restored according to eq. (4.4).

For the analytic computation of the MIs we follow the approach of ref. [34], which was inspired by refs. [54-56]. See also refs. [39, 57] for recent applications and some more details of this method. The basic strategy is to express each MI in terms of known integrals (with less propagators) and integrals that are quasi-finite in $d=4-2 \epsilon$ or higher, in practice $d=6-2 \epsilon, d=8-2 \epsilon$, or $d=10-2 \epsilon$ dimensions using dimensional recurrence relations [58-60] and IBP reduction. The integrands of quasi-finite integrals in the Feynman parameter representation are by definition free of endpoint singularities. For each MI we determine a suitable set of related quasi-finite integrals using the program Reduze2 [61]. We then expand their integrands and perform the integrations over the Feynman parameters order-by-order in $\epsilon$ using the Maple package HyperInt [62]. In order to deal with threshold singularities (related to the 'physical thresholds' discussed in section 3) HyperInt automatically performs a contour deformation by adding an infinitesimal imaginary part to one of the Feynman parameters. In general, this procedure leads to unreliable results for the imaginary parts of the MIs (for $\omega=-1$ ), which is however irrelevant for our calculation, because only the real parts contribute to our soft function. We discuss the evaluation of the three-loop integrals with non-zero imaginary parts in more detail in appendix D. To determine the required dimensional recurrence relations we use LiteRed [63, 64]. Via IBP reduction, we can bring the linear relations between the MIs (collected in the vector $\vec{I}$ ) in $d+2$ and $d$ dimensions into the form $\vec{I}(d+2)=\boldsymbol{A}(d) \cdot \vec{I}(d)$ for arbitrary $d$. We then obtain the analytic results for the MIs by solving the set of linear equations generated by (up to three) iterations of the real matrix $\boldsymbol{A}(d)$ with our results for the quasi-finite integrals (in $d=6-2 \epsilon, d=8-2 \epsilon$, and $d=10-2 \epsilon$ dimensions) as the input. Finally, we check the analytic expressions numerically using the sector decomposition program FIESTA4 [65].

\section{$5 \quad$ Results}

Evaluating all relevant three-loop Feynman diagrams as described in the previous section and summing up their contributions yields $\sigma(\omega)$ in eq. (3.11) at $\mathcal{O}\left(\alpha_{s}^{3}\right)$. According to

\footnotetext{
${ }^{10}$ Note that $S(\omega)$ vanishes for negative $\omega$. In view of eqs. (3.5) and (3.13) it is therefore natural to pull out a factor of $(-\omega)$, instead of $(+\omega)$, to the power determined by dimensional counting.
} 
eq. (3.5) and using eq. (3.13) we obtain the bare $\mathrm{N}^{3} \mathrm{LO}$ soft function ${ }^{11}$

$$
\begin{aligned}
S_{\eta}^{\text {bare }}(\omega)= & \delta(\omega)+\frac{Z_{\alpha} \alpha_{s}}{4 \pi} \frac{1}{\omega}\left(\frac{\mu}{\hat{\omega}}\right)^{2 \epsilon} C_{S_{\eta}} K_{S} \\
+ & \left(\frac{Z_{\alpha} \alpha_{s}}{4 \pi}\right)^{2} \frac{1}{\omega}\left(\frac{\mu}{\hat{\omega}}\right)^{4 \epsilon}\left(C_{S_{\eta}}^{2} K_{S S}+C_{S_{\eta}} C_{A} K_{S A}+C_{S_{\eta}} n_{f} T_{F} K_{S f}\right) \\
+ & \left(\frac{Z_{\alpha} \alpha_{s}}{4 \pi}\right)^{3} \frac{1}{\omega}\left(\frac{\mu}{\hat{\omega}}\right)^{6 \epsilon}\left[C_{S_{\eta}}^{3} K_{S S S}+C_{S_{\eta}}^{2} C_{A} K_{S S A}+C_{S_{\eta}} C_{A}^{2} K_{S A A}\right. \\
& +C_{S_{\eta}}^{2} n_{f} T_{F} K_{S S f}+C_{S_{\eta}} C_{F} n_{f} T_{F} K_{S F f}+C_{S_{\eta}} C_{A} n_{f} T_{F} K_{S A f} \\
& \left.+C_{S_{\eta}}\left(n_{f} T_{F}\right)^{2} K_{S f f}+\mathcal{C}_{3}^{\eta} K_{\mathcal{C}_{3}}\right]+\mathcal{O}\left(\alpha_{s}^{4}\right),
\end{aligned}
$$

where the coefficients $K_{X}$ are given in appendix B. The subscript $\eta=q \bar{q}, q g, g g$ indicates the partonic (production) channel. All dependence of the soft function on the kinematic variables $n_{12}, n_{1 J}$ and $n_{2 J}$ is encoded in

$$
\hat{\omega} \equiv \omega \sqrt{\frac{2 n_{12}}{n_{1 J} n_{2 J}}},
$$

which is invariant under the rescaling in eq. (4.1). This follows from the (re)scaling invariance of the Wilson lines in eq. (2.12). Following ref. [44] we have included a factor of $\sqrt{2}$ in the definition of $\hat{\omega}$ for convenience.

The color factor $C_{S_{\eta}}$ depends on the partonic channel and can be expressed in terms of the quadratic Casimir invariants $C_{F}=\left(N_{c}^{2}-1\right) /\left(2 N_{c}\right)$ and $C_{A}=N_{c}$ of $\mathrm{SU}\left(N_{c}\right)$ with $T_{F}=1 / 2[44]:$

$$
C_{S_{q \bar{q}}}=C_{F}-\frac{C_{A}}{2}, \quad C_{S_{q g}}=C_{S_{g g}}=\frac{C_{A}}{2} .
$$

The color factor $\mathcal{C}_{3}^{\eta}$ originates from tripole color structures that first appear at three loops and is computed below. The number of light (massless) quark flavors is denoted by $n_{f}$. For practical reasons our code performing the color algebra for the three-loop Feynman diagrams returns the color factors expressed in terms of $N_{c}$. Due to non-Abelian exponentiation $[66,67]$ and Casimir scaling of the color dipole contributions we are nevertheless able to uniquely reconstruct the color factors in eq. (5.1) in terms of Casimir invariants as explained in section 5.2 .

We define $\alpha_{s} \equiv \alpha_{s}(\mu)$ to be the renormalized QCD coupling constant. We stress that $Z_{\alpha} \mu^{2 \epsilon} \alpha_{s}\left(=\alpha_{s}^{\text {bare }}\right)$ and therefore the bare soft function is independent of the renormalization scale $\mu$. For renormalized quantities we use the $\overline{\mathrm{MS}}$ scheme throughout this work. The relevant terms of the strong coupling renormalization factor $Z_{\alpha}$ are

$$
Z_{\alpha}=1+\frac{\alpha_{s}}{4 \pi}\left(-\frac{\beta_{0}}{\epsilon}\right)+\left(\frac{\alpha_{s}}{4 \pi}\right)^{2}\left(\frac{\beta_{0}^{2}}{\epsilon^{2}}-\frac{\beta_{1}}{2 \epsilon}\right)+\mathcal{O}\left(\alpha_{s}^{3}\right)
$$

with

$$
\beta_{0}=\frac{11}{3} C_{A}-\frac{4}{3} T_{F} n_{f}, \quad \beta_{1}=\frac{34}{3} C_{A}^{2}-\frac{20}{3} C_{A} n_{f} T_{F}-4 C_{F} n_{f} T_{F} .
$$

\footnotetext{
${ }^{11}$ Here and in the following we assume $\omega>0$ and do not write out the $\theta(\omega)$ from eq. (3.13) for brevity.
} 
Following ref. [18] we conveniently perform the renormalization of the soft function in Laplace space. This is, because the Laplace transformation according to

$$
\tilde{s}_{\eta}(L)=\int_{0}^{\infty} \mathrm{d} \omega \exp \left(-\frac{\omega}{\kappa e^{\gamma_{E}}}\right) S_{\eta}(\omega)
$$

with

$$
L=\ln \left(\frac{\kappa}{\mu} \sqrt{\frac{2 n_{12}}{n_{1 J} n_{2 J}}}\right)
$$

and $\kappa$ as defined in eq. (2.6), turns the momentum space convolutions between renormalization factor, anomalous dimension, and renormalized soft function into simple (local) products, cf. eq. (2.5). The Laplace transform of the bare momentum space soft function in eq. (5.1) is obtained using the simple replacement rule

$$
\frac{1}{\omega}\left(\frac{\mu}{\hat{\omega}}\right)^{n \epsilon} \rightarrow e^{-n \epsilon\left(L+\gamma_{E}\right)} \Gamma(-n \epsilon) .
$$

\subsection{Anomalous dimension}

In Laplace space, the bare and renormalized soft function are related by the local renormalization factor $Z_{s_{\eta}}$,

$$
\tilde{s}_{\eta}^{\text {bare }}(\kappa)=Z_{s_{\eta}}(\mu) \tilde{s}_{\eta}(L, \mu) .
$$

The renormalized soft function obeys the RGE

$$
\frac{\mathrm{d}}{\mathrm{d} \ln \mu} \tilde{s}_{\eta}(L, \mu)=\Gamma^{S_{\eta}} \tilde{s}_{\eta}(L, \mu),
$$

with the soft anomalous dimension $\Gamma^{S_{\eta}}$. The Laplace space RGEs for the PDFs, the hard, and the jet function take the same form. RG invariance of the near-threshold cross section in eq. (2.5) implies $(\{a, b, c\}=\{q, \bar{q}, g\},\{q, g, q\}$, or $\{\bar{q}, g, \bar{q}\}$, or $\{g, g, g\}, \eta=a b)$

$$
\Gamma^{H_{\eta}}+\Gamma^{f_{a}}+\Gamma^{f_{b}}+\Gamma^{J_{c}}+\Gamma^{S_{\eta}}=0
$$

where $^{12}$

$$
\begin{aligned}
& \Gamma^{f_{i}}=2 \Gamma_{\text {cusp }}^{i}\left(\alpha_{s}\right) \ln \tau_{i}+2 \gamma^{f_{i}}\left(\alpha_{s}\right), \\
& \Gamma^{J_{i}}=-2 \Gamma_{\text {cusp }}^{i}\left(\alpha_{s}\right) \ln \left(\frac{Q^{2}}{\mu^{2}}\right)-2 \gamma^{J_{i}}\left(\alpha_{s}\right),
\end{aligned}
$$

are the ( $x \rightarrow 1$ threshold) PDF and jet function anomalous dimensions in Laplace space, respectively. The variables $Q^{2}$ and $\tau_{i}$ are given in eq. (2.6) and we conveniently define $\Gamma_{\text {cusp }}^{q}=C_{F} \gamma_{\text {cusp }}$ and $\Gamma_{\text {cusp }}^{g}=C_{A} \gamma_{\text {cusp }}$ for the universal cusp anomalous dimension.

The anomalous dimension of the hard function is denoted by $\Gamma^{H_{\eta}}$. For scattering amplitudes with three massless partons the corresponding operator in color space reads [69, 70]

$$
\boldsymbol{\Gamma}^{H}=2 \operatorname{Re}\left[\sum_{(i, j)} \frac{\boldsymbol{T}_{i} \cdot \boldsymbol{T}_{j}}{2} \gamma_{\text {cusp }}\left(\alpha_{s}\right) \ln \frac{\mu^{2}}{-s_{i j}}+\sum_{i} \gamma^{i}\left(\alpha_{s}\right) \mathbb{1}+f\left(\alpha_{s}\right) \sum_{(i, j, k)} \mathcal{T}_{i i j k}\right],
$$

\footnotetext{
${ }^{12}$ In this paper we use the same convention for $\gamma^{J_{i}}$ as refs. [18, 68]. In order to switch to the convention of refs. [34, 39] one has to multiply our $\gamma^{J_{i}}$ by $-1 / 2$.
} 
where $\boldsymbol{T}_{i}$ is the color charge operator for the $i$-th parton leg of the scattering amplitude defined in eq. (2.16) and $s_{i j} \equiv 2 \sigma_{i j} p_{i} \cdot p_{j}+i 0$, where the sign factor $\sigma_{i j}=+1$, if the momenta of $i$-th and $j$-th parton $p_{i}$ and $p_{j}$ are both incoming or outgoing, and $\sigma_{i j}=-1$ otherwise. The sums are over all combinations of distinct parton indices. The non-cusp anomalous dimension $\gamma^{i}$ is associated with each external massless (anti)quark $\left(\gamma^{i}=\gamma^{q}\right)$ or gluon $\left(\gamma^{i}=\gamma^{g}\right)$. The last term in eq. (5.14) corresponds to a color tripole contribution and is non-zero starting from three loops. The color structure $\mathcal{T}_{i j k l}$ is given by [69]

$$
\mathcal{T}_{i i j k}=\frac{1}{2} f^{a d e} f^{b c e}\left\{\boldsymbol{T}_{i}^{a}, \boldsymbol{T}_{i}^{b}\right\} \boldsymbol{T}_{j}^{c} \boldsymbol{T}_{k}^{d} .
$$

The three-loop contribution to the associated coefficient

$$
f\left(\alpha_{s}\right)=\sum_{n \geq 3}\left(\frac{\alpha_{s}}{4 \pi}\right)^{n} f_{n}
$$

was computed in ref. [71] and reads

$$
f_{3}=16\left(2 \zeta_{2} \zeta_{3}+\zeta_{5}\right)
$$

Evaluating

$$
\Gamma^{H_{\eta}}=\frac{\left\langle a_{1} a_{2} a_{J}\left|\boldsymbol{\Gamma}^{H}\right| a_{1} a_{2} a_{J}\right\rangle}{\left\langle a_{1} a_{2} a_{J} \mid a_{1} a_{2} a_{J}\right\rangle}
$$

with

$$
\mathcal{C}_{3}^{\eta} \equiv \frac{\left\langle a_{1} a_{2} a_{J}\left|\mathcal{T}_{i i j k}\right| a_{1} a_{2} a_{J}\right\rangle}{\left\langle a_{1} a_{2} a_{J} \mid a_{1} a_{2} a_{J}\right\rangle}
$$

we obtain from eq. (5.11)

$$
\Gamma^{S_{\eta}}=-4 \Gamma_{\text {cusp }}^{\eta}\left(\alpha_{s}\right) L-2 \gamma^{S_{\eta}}\left(\alpha_{s}\right)-2 \mathcal{C}_{3}^{\eta} f\left(\alpha_{s}\right)
$$

where $\gamma^{S_{\eta}}$ is the non-cusp piece of the soft anomalous dimension, and we have

$$
\begin{array}{lll}
\Gamma_{\text {cusp }}^{q \bar{q}}=C_{S_{q \bar{q}}} \gamma_{\text {cusp }}, & \gamma^{S_{q \bar{q}}}=2 \gamma^{q}+\gamma^{g}+2 \gamma^{f_{q}}-\gamma^{J_{g}}, & \mathcal{C}_{3}^{q \bar{q}}=-6 \frac{C_{4}(F, A)}{C_{F}}+\frac{C_{A}^{3}}{4}=-\frac{3}{2} N_{c}, \\
\Gamma_{\text {cusp }}^{q g}=C_{S_{q g}} \gamma_{\text {cusp }}, & \gamma^{S_{q g}}=2 \gamma^{q}+\gamma^{g}+\gamma^{f_{q}}+\gamma^{f_{g}}-\gamma^{J_{q}}, & \mathcal{C}_{3}^{q g}=-6 \frac{C_{4}(F, A)}{C_{F}}+\frac{C_{A}^{3}}{4}=-\frac{3}{2} N_{c}, \\
\Gamma_{\text {cusp }}^{g g}=C_{S_{g g}} \gamma_{\text {cusp }}, & \gamma^{S_{g g}}=3 \gamma^{g}+2 \gamma^{f_{g}}-\gamma^{J_{g}}, & \mathcal{C}_{3}^{g g}=-6 \frac{C_{4}(A, A)}{C_{A}}+\frac{C_{A}^{3}}{4}=-9 N_{c},
\end{array}
$$

for the different parton channels. In order to obtain the explicit expressions for the color factor $\mathcal{C}_{3}^{\eta}$ in terms of the quartic Casimir invariants

$$
C_{4}(R, A)=\frac{d_{R}^{a b c d} d_{A}^{a b c d}}{N_{R}}
$$

where $d_{R}^{a b c d}=\operatorname{tr}_{R}\left[T_{R}^{(a} T_{R}^{b} T_{R}^{c} T_{R}^{d)}\right]$ denotes the fully symmetric rank-four tensor of the $\mathrm{SU}\left(N_{c}\right)$ representation $R$, and $N_{A}=N_{c}^{2}-1, N_{F}=N_{c}$, we employed the color code [72]. All 
anomalous dimensions on the right-hand sides of eq. (5.21) are known at least to threeloop order, see appendix A. ${ }^{13}$ Just like the cusp also the non-cusp anomalous dimension $\gamma^{S_{\eta}}$ obeys Casimir scaling up to three loops, i.e.

$$
\frac{\gamma^{S_{q \bar{q}}}}{C_{S_{q \bar{q}}}}=\frac{\gamma^{S_{q g}}}{C_{S_{q g}}}=\frac{\gamma^{S_{g g}}}{C_{S_{g g}}} .
$$

Knowing $\Gamma^{S_{\eta}}$ we can determine the soft function renormalization factor $Z_{s_{\eta}}$ via $\Gamma^{S_{\eta}}=$ $-\mathrm{d} \ln Z_{s_{\eta}} / \mathrm{d} \ln \mu$. Up to three loops we find $[69,76]^{14}$

$$
\begin{aligned}
Z_{s}=1+\frac{\alpha_{s}}{4 \pi}\left(\frac{\Gamma_{0}^{S \prime}}{4 \epsilon^{2}}+\frac{\Gamma_{0}^{S}}{2 \epsilon}\right)+\left(\frac{\alpha_{s}}{4 \pi}\right)^{2}\left[\frac{\left(\Gamma_{0}^{S \prime}\right)^{2}}{32 \epsilon^{4}}+\frac{\Gamma_{0}^{S \prime}}{8 \epsilon^{3}}\left(\Gamma_{0}^{S}-\frac{3}{2} \beta_{0}\right)+\frac{\Gamma_{0}^{S}}{8 \epsilon^{2}}\left(\Gamma_{0}^{S}-2 \beta_{0}\right)+\frac{\Gamma_{1}^{S \prime}}{16 \epsilon^{2}}+\frac{\Gamma_{1}^{S}}{4 \epsilon}\right] \\
+\left(\frac{\alpha_{s}}{4 \pi}\right)^{3}\left[\frac{\left(\Gamma_{0}^{S \prime}\right)^{3}}{384 \epsilon^{6}}+\frac{\left(\Gamma_{0}^{S \prime}\right)^{2}}{64 \epsilon^{5}}\left(\Gamma_{0}^{S}-3 \beta_{0}\right)+\frac{\Gamma_{0}^{S \prime}}{32 \epsilon^{4}}\left(\Gamma_{0}^{S}-\frac{4}{3} \beta_{0}\right)\left(\Gamma_{0}^{S}-\frac{11}{3} \beta_{0}\right)+\frac{\Gamma_{0}^{S \prime} \Gamma_{1}^{S \prime}}{64 \epsilon^{4}}\right. \\
+\frac{\Gamma_{0}^{S}}{48 \epsilon^{3}}\left(\Gamma_{0}^{S}-2 \beta_{0}\right)\left(\Gamma_{0}^{S}-4 \beta_{0}\right)+\frac{\Gamma_{0}^{S \prime}}{16 \epsilon^{3}}\left(\Gamma_{1}^{S}-\frac{16}{9} \beta_{1}\right)+\frac{\Gamma_{1}^{S \prime}}{32 \epsilon^{3}}\left(\Gamma_{0}^{S}-\frac{20}{9} \beta_{0}\right) \\
\left.+\frac{\Gamma_{0}^{S} \Gamma_{1}^{S}}{8 \epsilon^{2}}-\frac{\beta_{0} \Gamma_{1}^{S}+\beta_{1} \Gamma_{0}^{S}}{6 \epsilon^{2}}+\frac{\Gamma_{2}^{S \prime}}{36 \epsilon^{2}}+\frac{\Gamma_{2}^{S}}{6 \epsilon}\right]+\mathcal{O}\left(\alpha_{s}^{4}\right),
\end{aligned}
$$

where

$$
\Gamma^{S \prime} \equiv \frac{\partial}{\partial \ln \mu} \Gamma^{S}
$$

and we have expanded the anomalous dimensions as

$$
\Gamma^{S}=\sum_{n=0}^{\infty} \Gamma_{n}^{S}\left(\frac{\alpha_{s}}{4 \pi}\right)^{n+1}, \quad \Gamma^{S \prime}=\sum_{n=0}^{\infty} \Gamma_{n}^{S \prime}\left(\frac{\alpha_{s}}{4 \pi}\right)^{n+1} .
$$

As expected, $Z_{s}$ absorbs all divergences of our explicit result for the bare Laplace space soft function $\tilde{s}(\kappa)$. This represents a strong check of our calculation and at the same time confirms the universal infrared structure of QCD scattering amplitudes with three massless parton legs, as predicted by eq. (5.14), at three loops.

\subsection{Renormalized results}

As mentioned above our results for the bare soft function in the different parton channels are initially expressed in terms of $N_{c}$. In order to rewrite the color factors in terms of (quadratic and quartic) Casimir invariants we proceed as follows. Non-Abelian exponentiation [66, 67] restricts the three-loop contribution to our soft function for all parton channels to be a universal linear combination of the color factors

$$
C_{S}^{3}, \quad C_{S}^{2} C_{A}, \quad C_{S} C_{A}^{2}, \quad C_{S}^{2} n_{f} T_{F}, \quad C_{S} C_{F} n_{f} T_{F}, \quad C_{S} C_{A} n_{f} T_{F}, \quad C_{S} n_{f}^{2} T_{F}^{2}, \quad \mathcal{C}_{3} .
$$

First, we discuss how to determine the coefficients of the color factors for $n_{f}=0$. For the $g g \rightarrow g$ channel we have according to eqs. (5.3) and (5.21) $C_{S}=N_{c} / 2$ and $\mathcal{C}_{3}=-9 N_{c}$. So,

\footnotetext{
${ }^{13}$ Starting from four loops the cusp and non-cusp anomalous dimensions violate Casimir scaling [73-75].

${ }^{14}$ For brevity we suppress the subscript $\eta$ of the soft function in following.
} 
all color factors except for $\mathcal{C}_{3}$ are proportional to $N_{c}^{3}$. We can therefore directly identify the color tripole contribution $\propto \mathcal{C}_{3}$ in our soft function result. Once this is fixed we take our result for the $q \bar{q} \rightarrow g$ channel and subtract the tripole term with $\mathcal{C}_{3}^{g g} \rightarrow \mathcal{C}_{3}^{q \bar{q}}$. The remaining terms in $S_{q \bar{q}}^{\text {bare }}$ can now be uniquely expressed in terms of the dipole-type color factors in eq. (5.27) using the replacement rules

$$
\frac{1}{N_{c}^{3}} \rightarrow-8 C_{S_{q \bar{q}}}^{3}, \quad \frac{1}{N_{c}} \rightarrow 4 C_{S_{q \bar{q}}}^{2} C_{A}, \quad N_{c} \rightarrow-2 C_{S_{q \bar{q}}} C_{A}^{2} .
$$

Concerning the color factors involving $n_{f}$, we first map the $C_{S} C_{F} n_{f} T_{F}$ contribution to the $n_{f} T_{F} N_{c}^{0}$ term in our result for $S_{g g}^{\text {bare }}$. After that we can rewrite the remaining $n_{f}$ dependent terms in $S_{q \bar{q}}^{\text {bare }}$ by replacing

$$
\frac{n_{f} T_{F}}{N_{c}^{2}} \rightarrow 4 C_{S_{q \bar{q}}}^{2} n_{f} T_{F}, \quad n_{f} T_{F} N_{c}^{0} \rightarrow-2 C_{S_{q \bar{q}}} C_{A} n_{f} T_{F}, \quad \frac{n_{f}^{2} T_{F}^{2}}{N_{c}} \rightarrow-2 C_{S_{q \bar{q}}} n_{f}^{2} T_{F}^{2} .
$$

In this way $S_{q \bar{q}}^{\text {bare }}$ is completely expressed in terms of Casimir invariants and matches eq. (5.1). We successfully checked the universality of this expression against our explicit results in terms of $N_{c}$ for $S_{q g}^{\text {bare }}$ and $S_{g g}^{\text {bare }}$ by adjusting $C_{S}$ and $\mathcal{C}_{3}$ according to eqs. (5.3) and (5.21).

Solving the RGE in eq. (5.10) with the anomalous dimension in eq. (5.20), the renormalized soft function in Laplace space can be written as

$$
\begin{gathered}
\tilde{s}(L, \mu)=1+\frac{\alpha_{s}}{4 \pi}\left[2 \Gamma_{0} L^{2}+2 \gamma_{0}^{S} L+c_{1}^{S}\right]+\left(\frac{\alpha_{s}}{4 \pi}\right)^{2}\left[2 \Gamma_{0}^{2} L^{4}-\frac{4}{3} \Gamma_{0}\left(\beta_{0}-3 \gamma_{0}^{S}\right) L^{3}\right. \\
\left.+2\left(\Gamma_{0} c_{1}^{S}+\Gamma_{1}-\beta_{0} \gamma_{0}^{S}+\left(\gamma_{0}^{S}\right)^{2}\right) L^{2}+2\left(c_{1}^{S}\left(\gamma_{0}^{S}-\beta_{0}\right)+\gamma_{1}^{S}\right) L+c_{2}^{S}\right] \\
+\left(\frac{\alpha_{s}}{4 \pi}\right)^{3}\left[\frac{4 \Gamma_{0}^{3}}{3} L^{6}+\frac{4 \Gamma_{0}^{2}}{3}\left(3 \gamma_{0}^{S}-2 \beta_{0}\right) L^{5}+\frac{2 \Gamma_{0}}{3}\left(2 \beta_{0}^{2}+3 \Gamma_{0} c_{1}^{S}+6 \Gamma_{1}+6\left(\gamma_{0}^{S}\right)^{2}\right.\right. \\
\left.-10 \beta_{0} \gamma_{0}^{S}\right) L^{4}+\frac{4}{3}\left(3 \gamma_{0}^{S}\left(\Gamma_{0} c_{1}^{S}+\Gamma_{1}\right)-\beta_{0}\left(4 \Gamma_{0} c_{1}^{S}+2 \Gamma_{1}+3\left(\gamma_{0}^{S}\right)^{2}\right)\right. \\
\left.-\Gamma_{0}\left(\beta_{1}-3 \gamma_{1}^{S}\right)+2 \beta_{0}^{2} \gamma_{0}^{S}+\left(\gamma_{0}^{S}\right)^{3}\right) L^{3}+2\left(\Gamma_{0} c_{2}^{S}+\Gamma_{2}+2 \gamma_{0}^{S} \gamma_{1}^{S}\right. \\
\left.+c_{1}^{S}\left(2 \beta_{0}^{2}+\Gamma_{1}-3 \beta_{0} \gamma_{0}^{S}+\left(\gamma_{0}^{S}\right)^{2}\right)-\beta_{1} \gamma_{0}^{S}-2 \beta_{0} \gamma_{1}^{S}\right) L^{2} \\
\left.+2\left(c_{1}^{S}\left(\gamma_{1}^{S}-\beta_{1}\right)+c_{2}^{S}\left(\gamma_{0}^{S}-2 \beta_{0}\right)+\gamma_{2}^{S}+\mathcal{C}_{3} f_{3}\right) L+c_{3}^{S}\right] .
\end{gathered}
$$

Here we have expanded the anomalous dimensions in eq. (5.21) as

$$
\Gamma_{\text {cusp }}=\sum_{n=0}^{\infty} \Gamma_{n}\left(\frac{\alpha_{s}}{4 \pi}\right)^{n+1}, \quad \gamma^{S}=\sum_{n=0}^{\infty} \gamma_{n}^{S}\left(\frac{\alpha_{s}}{4 \pi}\right)^{n+1} .
$$


Our calculation determines the non-logarithmic terms in eq. (5.30) to be

$$
\begin{aligned}
c_{1}^{S}= & C_{S} \pi^{2}, \\
c_{2}^{S}= & C_{S}^{2} \frac{\pi^{4}}{2}+C_{S} C_{A}\left(-\frac{22 \zeta_{3}}{9}-\frac{14 \pi^{4}}{15}+\frac{670 \pi^{2}}{108}+\frac{2428}{81}\right)+C_{S} n_{f} T_{F}\left(\frac{8 \zeta_{3}}{9}-\frac{50 \pi^{2}}{27}-\frac{656}{81}\right), \\
c_{3}^{S}= & C_{S}^{3} \frac{\pi^{6}}{6}+C_{S}^{2} C_{A}\left(-\frac{22}{9} \pi^{2} \zeta_{3}-\frac{14 \pi^{6}}{15}+\frac{335 \pi^{4}}{54}+\frac{2428 \pi^{2}}{81}\right)+C_{S} C_{A}^{2}\left(\frac{1108 \zeta_{3}^{2}}{9}-\frac{242 \pi^{2} \zeta_{3}}{27}\right. \\
& \left.\quad-\frac{87052 \zeta_{3}}{243}-\frac{572 \zeta_{5}}{9}+\frac{65333 \pi^{6}}{51030}-\frac{26153 \pi^{4}}{2430}+\frac{256739 \pi^{2}}{4374}+\frac{5211949}{13122}\right) \\
& +C_{S} C_{F} n_{f} T_{F}\left(\frac{112 \pi^{2} \zeta_{3}}{9}+\frac{5680 \zeta_{3}}{81}+\frac{448 \zeta_{5}}{9}+\frac{152 \pi^{4}}{405}-\frac{385 \pi^{2}}{27}-\frac{42727}{243}\right) \\
& +C_{S} C_{A} n_{f} T_{F}\left(-\frac{248}{27} \pi^{2} \zeta_{3}+\frac{2432 \zeta_{3}}{81}-\frac{80 \zeta_{5}}{3}+\frac{464 \pi^{4}}{243}-\frac{69254 \pi^{2}}{2187}-\frac{825530}{6561}\right) \\
& +C_{S}^{2} n_{f} T_{F}\left(\frac{8 \pi^{2} \zeta_{3}}{9}-\frac{50 \pi^{4}}{27}-\frac{656 \pi^{2}}{81}\right)+C_{S} n_{f}^{2} T_{F}^{2}\left(\frac{3520 \zeta_{3}}{243}+\frac{88 \pi^{4}}{243}+\frac{784 \pi^{2}}{243}-\frac{1024}{6561}\right) \\
& +2 \mathcal{C}_{3}\left(\frac{40 \zeta_{3}^{2}}{3}-\frac{1031 \pi^{6}}{5670}\right),
\end{aligned}
$$

The expression for $c_{3}^{S}$ is new and represents the main result of this work.

For completeness we also give here the renormalized soft function in momentum space

$$
S(\omega, \mu)=\sum_{m=0}^{\infty}\left(\frac{\alpha_{s}}{4 \pi}\right)^{m} S^{(m)}(\omega, \mu)
$$

obtained from inverting eq. (5.6). The coefficients in the $\alpha_{s}$ expansion take the form

$$
S^{(m)}(\omega, \mu)=S_{-1}^{(m)} \delta(\omega)+\sum_{n=0}^{2 m-1} S_{n}^{(m)} \hat{\mathcal{L}}_{n}(\omega)
$$

with the plus distributions

$$
\hat{\mathcal{L}}_{n}(\omega)=\left[\frac{\theta(\omega)}{\omega} \ln ^{n}\left(\frac{\hat{\omega}}{\mu}\right)\right]_{+}=\lim _{\varepsilon \rightarrow 0} \frac{\mathrm{d}}{\mathrm{d} \omega}\left[\theta(\omega-\varepsilon) \frac{\ln ^{n+1}\left(\frac{\hat{\omega}}{\mu}\right)}{n+1}\right] .
$$

The constants $S_{n}^{(m)}$ can be expressed in terms of anomalous dimension coefficients and the 
$c_{i}^{S}$ in eq. (5.32):

$$
\begin{aligned}
& S_{1}^{(1)}=4 \Gamma_{0}, \\
& S_{0}^{(1)}=2 \gamma_{0}^{S} \text {, } \\
& S_{-1}^{(1)}=-\frac{\pi^{2}}{3} \Gamma_{0}+c_{1}^{S}, \\
& S_{3}^{(2)}=8 \Gamma_{0}^{2} \text {, } \\
& S_{2}^{(2)}=4 \Gamma_{0}\left(3 \gamma_{0}^{S}-\beta_{0}\right) \text {, } \\
& S_{1}^{(2)}=4 \Gamma_{1}+4 \Gamma_{0}\left(c_{1}^{S}-\pi^{2} \Gamma_{0}\right)+4 \gamma_{0}^{S}\left(\gamma_{0}^{S}-\beta_{0}\right), \\
& S_{0}^{(2)}=2 \Gamma_{0}\left(8 \zeta_{3} \Gamma_{0}+\frac{\pi^{2}}{3} \beta_{0}-\pi^{2} \gamma_{0}^{S}\right)+2 \gamma_{1}^{S}+2 c_{1}^{S}\left(\gamma_{0}^{S}-\beta_{0}\right) \text {, } \\
& S_{-1}^{(2)}=\frac{\pi^{4}}{30} \Gamma_{0}^{2}-\frac{\pi^{2}}{3} \Gamma_{1}+8 \zeta_{3} \Gamma_{0}\left(\gamma_{0}^{S}-\frac{\beta_{0}}{3}\right)+\frac{\pi^{2}}{3} \gamma_{0}^{S}\left(\beta_{0}-\gamma_{0}^{S}\right)-\frac{\pi^{2}}{3} \Gamma_{0} c_{1}^{S}+c_{2}^{S}, \\
& S_{5}^{(3)}=8 \Gamma_{0}^{3} \text {, } \\
& S_{4}^{(3)}=20 \Gamma_{0}^{2}\left(\gamma_{0}^{S}-\frac{2}{3} \beta_{0}\right) \text {, } \\
& S_{3}^{(3)}=16 \Gamma_{0}\left[\frac{\beta_{0}^{2}}{3}+\Gamma_{1}+\gamma_{0}^{S}\left(\gamma_{0}^{S}-\frac{5}{3} \beta_{0}\right)\right]+8 \Gamma_{0}^{2}\left(c_{1}^{S}-\frac{5 \pi^{2}}{3} \Gamma_{0}\right), \\
& S_{2}^{(3)}=20 \Gamma_{0}^{2}\left[8 \zeta_{3} \Gamma_{0}+\pi^{2}\left(\frac{2}{3} \beta_{0}-\gamma_{0}^{S}\right)\right]+4 \Gamma_{0}\left[3 \gamma_{1}^{S}-\beta_{1}+c_{1}^{S}\left(3 \gamma_{0}^{S}-4 \beta_{0}\right)\right]+4\left(\gamma_{0}^{S}\right)^{3} \\
& +4\left(\beta_{0} \gamma_{0}^{S}-\Gamma_{1}\right)\left(2 \beta_{0}-3 \gamma_{0}^{S}\right) \\
& S_{1}^{(3)}=4 \Gamma_{2}+\frac{2 \pi^{4}}{3} \Gamma_{0}^{3}+160 \zeta_{3} \Gamma_{0}^{2}\left(\gamma_{0}^{S}-\frac{2 \beta_{0}}{3}\right)+8 \pi^{2} \Gamma_{0}\left[-\frac{\beta_{0}^{2}}{3}-\Gamma_{1}+\frac{5}{3} \beta_{0} \gamma_{0}^{S}-\left(\gamma_{0}^{S}\right)^{2}\right] \\
& -8 \beta_{0} \gamma_{1}^{S}+4 \gamma_{0}^{S}\left(2 \gamma_{1}^{S}-\beta_{1}\right)+4 c_{1}^{S}\left[\left(2 \beta_{0}-\gamma_{0}^{S}\right)\left(\beta_{0}-\gamma_{0}^{S}\right)-\pi^{2} \Gamma_{0}^{2}+\Gamma_{1}\right]+4 \Gamma_{0} c_{2}^{S}, \\
& S_{0}^{(3)}=16 \Gamma_{0}^{3}\left(12 \zeta_{5}-\frac{5 \pi^{2} \zeta_{3}}{3}\right)+\frac{\pi^{4}}{3} \Gamma_{0}^{2}\left(\gamma_{0}^{S}-\frac{2}{3} \beta_{0}\right)+2 \pi^{2} \Gamma_{1}\left(\frac{2}{3} \beta_{0}-\gamma_{0}^{S}\right)+2 \pi^{2} \Gamma_{0}\left(\frac{\beta_{1}}{3}-\gamma_{1}^{S}\right) \\
& +32 \zeta_{3} \Gamma_{0}\left[\frac{\beta_{0}^{2}}{3}+\Gamma_{1}-\frac{5}{3} \beta_{0} \gamma_{0}^{S}+\left(\gamma_{0}^{S}\right)^{2}\right]+2 \gamma_{2}^{S}-\frac{2 \pi^{2}}{3} \gamma_{0}^{S}\left(2 \beta_{0}-\gamma_{0}^{S}\right)\left(\beta_{0}-\gamma_{0}^{S}\right) \\
& +2 c_{1}^{S}\left[-\beta_{1}+8 \zeta_{3} \Gamma_{0}^{2}+\pi^{2} \Gamma_{0}\left(\frac{4}{3} \beta_{0}-\gamma_{0}^{S}\right)+\gamma_{1}^{S}\right]+2 c_{2}^{S}\left(\gamma_{0}^{S}-2 \beta_{0}\right)+2 \mathcal{C}_{3} f_{3}, \\
& S_{-1}^{(3)}=\frac{5}{3} \Gamma_{0}^{3}\left(32 \zeta_{3}^{2}-\frac{\pi^{6}}{42}\right)+\Gamma_{0}^{2}\left(\frac{40 \pi^{2} \zeta_{3}}{3}-96 \zeta_{5}\right)\left(\frac{2}{3} \beta_{0}-\gamma_{0}^{S}\right)+8 \zeta_{3} \Gamma_{1}\left(\gamma_{0}^{S}-\frac{2}{3} \beta_{0}\right) \\
& -\frac{\pi^{2}}{3} \Gamma_{2}+\Gamma_{0}\left[\frac{\pi^{4}}{45}\left(\beta_{0}^{2}-5 \beta_{0} \gamma_{0}^{S}+3\left(\gamma_{0}^{S}\right)^{2}+3 \Gamma_{1}\right)+8 \zeta_{3}\left(\gamma_{1}^{S}-\frac{\beta_{1}}{3}\right)\right] \\
& +\frac{8 \zeta_{3}}{3} \gamma_{0}^{S}\left(2 \beta_{0}-\gamma_{0}^{S}\right)\left(\beta_{0}-\gamma_{0}^{S}\right)+\frac{\pi^{2}}{3} \gamma_{0}^{S}\left(\beta_{1}-2 \gamma_{1}^{S}\right)+\frac{2 \pi^{2}}{3} \beta_{0} \gamma_{1}^{S}-\frac{\pi^{2}}{3} \Gamma_{0} c_{2}^{S}+c_{3}^{S} \\
& +c_{1}^{S}\left[\frac{\pi^{4}}{30} \Gamma_{0}^{2}-\frac{\pi^{2}}{3} \Gamma_{1}+8 \zeta_{3} \Gamma_{0}\left(\gamma_{0}^{S}-\frac{4}{3} \beta_{0}\right)-\frac{\pi^{2}}{3}\left(2 \beta_{0}-\gamma_{0}^{S}\right)\left(\beta_{0}-\gamma_{0}^{S}\right)\right] .
\end{aligned}
$$




\section{Conclusion}

We have calculated the universal three-loop soft function contributing to factorized cross sections for EW boson production in the (threshold) limit, where the transverse momentum of the boson is close to its kinematically allowed maximum for a given (not too large) rapidity. To the best of our knowledge this represents the first result of a soft function for a three-parton process at three loops. We have derived a novel expression for the soft function in terms of a forward-scattering-type matrix element of Wilson line operators. This allowed us to avoid any phase space integrations and to straight-forwardly take advantage of well-established multi-loop technology in our calculation.

We present our results for the renormalized soft function both in Laplace space and momentum space in section 5.2. The three-loop non-logarithmic terms in eq. (5.32) represent the genuinely new information at this order. Our explicit three-loop calculation validates the relation between the anomalous dimensions of the PDFs and the corresponding hard, jet, and soft functions inferred from RG consistency of the near-threshold cross section. In this way we also confirm the nonzero (Casimir scaling violating) color tripole contribution to the three-loop soft and corresponding hard anomalous dimensions found in ref. [71].

The threshold approximation of the EW boson production cross section is strictly valid when the invariant mass of the hadronic radiation (including the proton remnants) is small compared to the transverse momentum of the boson. Phenomenologically, however, it usually performs well even far away from this limit, see e.g. refs. [25, 26]. Together with the three-loop jet function $[34,35]$ and the yet-unknown three-loop hard function our soft function result will allow to determine the $\mathrm{N}^{3} \mathrm{LO}$ corrections to the transversemomentum spectra of $\gamma, W^{ \pm}, Z$ and Higgs bosons in the (far-)tail region. Furthermore, it is necessary to carry out the resummation of threshold logarithms to $\mathrm{N}^{3} \mathrm{LL}^{\prime}$ (or $\mathrm{N}^{4} \mathrm{LL}$ ) accuracy. Once the QCD three-loop virtual corrections to the $q \bar{q} \rightarrow Y g$ (or crossingsymmetric) and $g g \rightarrow H g$ scattering amplitudes, i.e. the hard functions, become available, this may constitute significant progress in the precision phenomenology for Higgs, photon and EW gauge boson production in association with a jet at the LHC.

\section{Acknowledgments}

Z.L.L. is grateful to Thomas Becher, Matthias Neubert and Ding Yu Shao for valuable discussions. M.S. thanks Erik Panzer for inspiring feedback and explanations regarding HyperInt. We are indebted to Robin Brüser for providing the codes to perform the diagram generation, topology mapping, and partial fractioning and for his help with the color code. This research has been supported by the Cluster of Excellence PRISMA* (project ID 39083149), funded by the German Research Foundation (DFG). The research of Z.L.L. is supported by the U.S. Department of Energy under Contract No. DE-AC52-06NA25396, the LANL/LDRD program and within the framework of the TMD Topical Collaboration. All graphs were drawn using JaxoDraw [77]. 


\section{A Three-loop anomalous dimensions}

For completeness we list here the explicit expressions for all anomalous dimensions in eq. (5.21) to three-loop order. The convention for the loop expansions is analogous to eqs. (5.26) and (5.31). The coefficients of the cusp anomalous dimension are [78, 79]

$$
\begin{aligned}
\gamma_{0}^{\text {cusp }}= & 4, \\
\gamma_{1}^{\text {cusp }}= & \left(\frac{268}{9}-\frac{4 \pi^{2}}{3}\right) C_{A}-\frac{80}{9} T_{F} n_{f}, \\
\gamma_{2}^{\text {cusp }}= & C_{A}^{2}\left(\frac{490}{3}-\frac{536 \pi^{2}}{27}+\frac{44 \pi^{4}}{45}+\frac{88}{3} \zeta_{3}\right)+C_{A} T_{F} n_{f}\left(-\frac{1672}{27}+\frac{160 \pi^{2}}{27}-\frac{224}{3} \zeta_{3}\right) \\
& +C_{F} T_{F} n_{f}\left(-\frac{220}{3}+64 \zeta_{3}\right)-\frac{64}{27} T_{F}^{2} n_{f}^{2} .
\end{aligned}
$$

The coefficients of the soft non-cusp anomalous dimension $\gamma^{S}$ are [18]

$$
\begin{aligned}
\gamma_{0}^{S}=0 & \\
\gamma_{1}^{S}=C_{S} & {\left[C_{A}\left(28 \zeta_{3}+\frac{11 \pi^{2}}{9}-\frac{808}{27}\right)+n_{f} T_{F}\left(\frac{224}{27}-\frac{4 \pi^{2}}{9}\right)\right] } \\
\gamma_{2}^{S}=C_{S} & {\left[C_{A}^{2}\left(-\frac{88}{9} \pi^{2} \zeta_{3}+\frac{1316 \zeta_{3}}{3}-192 \zeta_{5}-\frac{88 \pi^{4}}{45}+\frac{6325 \pi^{2}}{243}-\frac{136781}{729}\right)\right.} \\
& +C_{A} n_{f} T_{F}\left(-\frac{1456 \zeta_{3}}{27}+\frac{16 \pi^{4}}{15}-\frac{2828 \pi^{2}}{243}+\frac{23684}{729}\right) \\
& \left.+C_{F} n_{f} T_{F}\left(\frac{3422}{27}-\frac{4 \pi^{2}}{3}-\frac{608 \zeta_{3}}{9}-\frac{16 \pi^{4}}{45}\right)+n_{f}^{2} T_{F}^{2}\left(\frac{8320}{729}+\frac{80 \pi^{2}}{81}-\frac{448 \zeta_{3}}{27}\right)\right]
\end{aligned}
$$

The hard anomalous dimensions $\gamma^{q}$ and $\gamma^{g}$ can be determined [69] from the divergent part of the on-shell quark and gluon form factors in QCD [80, 81] and read up to three loops

$$
\begin{aligned}
\gamma_{0}^{q}= & -3 C_{F} \\
\gamma_{1}^{q}= & C_{F}^{2}\left(-\frac{3}{2}+2 \pi^{2}-24 \zeta_{3}\right)+C_{F} C_{A}\left(-\frac{961}{54}-\frac{11 \pi^{2}}{6}+26 \zeta_{3}\right) \\
& +C_{F} T_{F} n_{f}\left(\frac{130}{27}+\frac{2 \pi^{2}}{3}\right), \\
\gamma_{2}^{q}= & C_{F}^{3}\left(-\frac{29}{2}-3 \pi^{2}-\frac{8 \pi^{4}}{5}-68 \zeta_{3}+\frac{16 \pi^{2}}{3} \zeta_{3}+240 \zeta_{5}\right) \\
& +C_{F}^{2} C_{A}\left(-\frac{151}{4}+\frac{205 \pi^{2}}{9}+\frac{247 \pi^{4}}{135}-\frac{844}{3} \zeta_{3}-\frac{8 \pi^{2}}{3} \zeta_{3}-120 \zeta_{5}\right) \\
& +C_{F} C_{A}^{2}\left(-\frac{139345}{2916}-\frac{7163 \pi^{2}}{486}-\frac{83 \pi^{4}}{90}+\frac{3526}{9} \zeta_{3}-\frac{44 \pi^{2}}{9} \zeta_{3}-136 \zeta_{5}\right) \\
& +C_{F}^{2} T_{F} n_{f}\left(\frac{2953}{27}-\frac{26 \pi^{2}}{9}-\frac{28 \pi^{4}}{27}+\frac{512}{9} \zeta_{3}\right)
\end{aligned}
$$




$$
\begin{aligned}
& +C_{F} C_{A} T_{F} n_{f}\left(-\frac{17318}{729}+\frac{2594 \pi^{2}}{243}+\frac{22 \pi^{4}}{45}-\frac{1928}{27} \zeta_{3}\right) \\
& +C_{F} T_{F}^{2} n_{f}^{2}\left(\frac{9668}{729}-\frac{40 \pi^{2}}{27}-\frac{32}{27} \zeta_{3}\right), \\
\gamma_{0}^{g}= & -\beta_{0}=-\frac{11}{3} C_{A}+\frac{4}{3} T_{F} n_{f}, \\
\gamma_{1}^{g}= & C_{A}^{2}\left(-\frac{692}{27}+\frac{11 \pi^{2}}{18}+2 \zeta_{3}\right)+C_{A} T_{F} n_{f}\left(\frac{256}{27}-\frac{2 \pi^{2}}{9}\right)+4 C_{F} T_{F} n_{f}, \\
\gamma_{2}^{g}= & C_{A}^{3}\left(-\frac{97186}{729}+\frac{6109 \pi^{2}}{486}-\frac{319 \pi^{4}}{270}+\frac{122}{3} \zeta_{3}-\frac{20 \pi^{2}}{9} \zeta_{3}-16 \zeta_{5}\right) \\
& +C_{A}^{2} T_{F} n_{f}\left(\frac{30715}{729}-\frac{1198 \pi^{2}}{243}+\frac{82 \pi^{4}}{135}+\frac{712}{27} \zeta_{3}\right) \\
& +C_{A} C_{F} T_{F} n_{f}\left(\frac{2434}{27}-\frac{2 \pi^{2}}{3}-\frac{8 \pi^{4}}{45}-\frac{304}{9} \zeta_{3}\right)-2 C_{F}^{2} T_{F} n_{f} \\
& +C_{A} T_{F}^{2} n_{f}^{2}\left(-\frac{538}{729}+\frac{40 \pi^{2}}{81}-\frac{224}{27} \zeta_{3}\right)-\frac{44}{9} C_{F} T_{F}^{2} n_{f}^{2} .
\end{aligned}
$$

At three loops the anomalous dimensions of the quark and gluon jet functions were derived in refs. [18, 68] based on RG consistency arguments and confirmed by the jet function calculations in refs. $[34,35]$. Their coefficients are

$$
\begin{aligned}
\gamma_{0}^{J_{q}}= & -3 C_{F} \\
\gamma_{1}^{J_{q}}= & C_{F}^{2}\left(-\frac{3}{2}+2 \pi^{2}-24 \zeta_{3}\right)+C_{F} C_{A}\left(-\frac{1769}{54}-\frac{11 \pi^{2}}{9}+40 \zeta_{3}\right) \\
& +C_{F} T_{F} n_{f}\left(\frac{242}{27}+\frac{4 \pi^{2}}{9}\right), \\
\gamma_{2}^{J_{q}}= & C_{F}^{3}\left(-\frac{29}{2}-3 \pi^{2}-\frac{8 \pi^{4}}{5}-68 \zeta_{3}+\frac{16 \pi^{2}}{3} \zeta_{3}+240 \zeta_{5}\right) \\
& +C_{F}^{2} C_{A}\left(-\frac{151}{4}+\frac{205 \pi^{2}}{9}+\frac{247 \pi^{4}}{135}-\frac{844}{3} \zeta_{3}-\frac{8 \pi^{2}}{3} \zeta_{3}-120 \zeta_{5}\right) \\
& +C_{F} C_{A}^{2}\left(-\frac{412907}{2916}-\frac{419 \pi^{2}}{243}-\frac{19 \pi^{4}}{10}+\frac{5500}{9} \zeta_{3}-\frac{88 \pi^{2}}{9} \zeta_{3}-232 \zeta_{5}\right) \\
& +C_{F}^{2} T_{F} n_{f}\left(\frac{4664}{27}-\frac{32 \pi^{2}}{9}-\frac{164 \pi^{4}}{135}+\frac{208}{9} \zeta_{3}\right) \\
& +C_{F} C_{A} T_{F} n_{f}\left(-\frac{5476}{729}+\frac{1180 \pi^{2}}{243}+\frac{46 \pi^{4}}{45}-\frac{2656}{27} \zeta_{3}\right) \\
& +C_{F} T_{F}^{2} n_{f}^{2}\left(\frac{13828}{729}-\frac{80 \pi^{2}}{81}-\frac{256}{27} \zeta_{3}\right),
\end{aligned}
$$


and

$$
\begin{aligned}
\gamma_{0}^{J_{g}}= & -\beta_{0} \\
\gamma_{1}^{J_{g}}= & C_{A}^{2}\left(-\frac{1096}{27}+\frac{11 \pi^{2}}{9}+16 \zeta_{3}\right)+C_{A} n_{f} T_{F}\left(\frac{368}{27}-\frac{4 \pi^{2}}{9}\right)+4 C_{F} T_{F} n_{f} \\
\gamma_{2}^{J_{g}}= & \left(-\frac{331153}{1458}+\frac{6217 \pi^{2}}{243}+260 \zeta_{3}-\frac{583 \pi^{4}}{270}-\frac{64 \pi^{2} \zeta_{3}}{9}-112 \zeta_{5}\right) C_{A}^{3} \\
& +\left(\frac{42557}{729}-\frac{2612}{243}-\frac{16 \zeta_{3}}{27}+\frac{154 \pi^{4}}{135}\right) C_{A}^{2} n_{f} T_{F}+\left(\frac{3622}{729}+\frac{80 \pi^{2}}{81}-\frac{448 \zeta_{3}}{27}\right) C_{A} n_{f}^{2} T_{F}^{2} \\
& +\left(\frac{4145}{27}-\frac{4 \pi^{2}}{3}-\frac{608 \zeta_{3}}{9}-\frac{16 \pi^{4}}{45}\right) C_{A} C_{F} n_{f} T_{F}-2 C_{F}^{2} n_{f} T_{F}-\frac{44}{9} C_{F} n_{F}^{2} T_{F}^{2}
\end{aligned}
$$

respectively. The anomalous dimensions describing the evolution of the quark and gluon PDFs near $x \rightarrow 1$ can be determined from the QCD splitting functions [79, 82] and were extracted in refs. $[83,84]$ up to three-loop order:

$$
\begin{aligned}
\gamma_{0}^{f_{q}}= & 3 C_{F} \\
\gamma_{1}^{f_{q}}= & C_{F}^{2}\left(\frac{3}{2}-2 \pi^{2}+24 \zeta_{3}\right)+C_{F} C_{A}\left(\frac{17}{6}+\frac{22 \pi^{2}}{9}-12 \zeta_{3}\right) \\
& -C_{F} T_{F} n_{f}\left(\frac{2}{3}+\frac{8 \pi^{2}}{9}\right) \\
\gamma_{2}^{f_{q}}= & C_{F}^{3}\left(\frac{29}{2}+3 \pi^{2}+\frac{8 \pi^{4}}{5}+68 \zeta_{3}-\frac{16 \pi^{2}}{3} \zeta_{3}-240 \zeta_{5}\right) \\
& +C_{F}^{2} C_{A}\left(\frac{151}{4}-\frac{205 \pi^{2}}{9}-\frac{247 \pi^{4}}{135}+\frac{844}{3} \zeta_{3}+\frac{8 \pi^{2}}{3} \zeta_{3}+120 \zeta_{5}\right) \\
& +C_{F}^{2} T_{F} n_{f}\left(-46+\frac{20 \pi^{2}}{9}+\frac{116 \pi^{4}}{135}-\frac{272}{3} \zeta_{3}\right) \\
& +C_{F} C_{A}^{2}\left(-\frac{1657}{36}+\frac{2248 \pi^{2}}{81}-\frac{\pi^{4}}{18}-\frac{1552}{9} \zeta_{3}+40 \zeta_{5}\right) \\
& +C_{F} C_{A} T_{F} n_{f}\left(40-\frac{1336 \pi^{2}}{81}+\frac{2 \pi^{4}}{45}+\frac{400}{9} \zeta_{3}\right) \\
& +C_{F} T_{F}^{2} n_{f}^{2}\left(-\frac{68}{9}+\frac{160 \pi^{2}}{81}-\frac{64}{9} \zeta_{3}\right) \\
\gamma_{0}^{f_{g}}= & \frac{11}{3} C_{A}-\frac{4}{3} T_{F} n_{f}=\beta_{0}, \\
\gamma_{1}^{f_{g}}= & 4 C_{A}^{2}\left(\frac{8}{3}+3 \zeta_{3}\right)-\frac{16}{3} C_{A} T_{F} n_{f}-4 C_{F} T_{F} n_{f},
\end{aligned}
$$




$$
\begin{aligned}
\gamma_{2}^{f_{g}}= & C_{A}^{3}\left[\frac{79}{2}+\frac{4 \pi^{2}}{9}+\frac{11 \pi^{4}}{54}+\left(\frac{536}{3}-\frac{8 \pi^{2}}{3}\right) \zeta_{3}-80 \zeta_{5}\right] \\
& -C_{A}^{2} T_{F} n_{f}\left(\frac{233}{9}+\frac{8 \pi^{2}}{9}+\frac{2 \pi^{4}}{27}+\frac{160}{3} \zeta_{3}\right) \\
& -\frac{241}{9} C_{A} C_{F} T_{F} n_{f}+2 C_{F}^{2} T_{F} n_{f}+\frac{58}{9} C_{A} T_{F}^{2} n_{f}^{2}+\frac{44}{9} C_{F} T_{F}^{2} n_{f}^{2}
\end{aligned}
$$

\section{B Bare data}

Here we present our expressions for the coefficients of the different color structures in the bare soft function, eq. (5.1). We show the results as an expansion in $\epsilon=(4-d) / 2$ to the order required for the calculation of the renormalized three-loop soft function:

$$
\begin{aligned}
& K_{S}=-\frac{8}{\epsilon}+\frac{2 \pi^{2} \epsilon}{3}+\frac{56 \zeta_{3} \epsilon^{2}}{3}+\frac{47 \pi^{4} \epsilon^{3}}{180}+\left(\frac{248 \zeta_{5}}{5}-\frac{14 \pi^{2} \zeta_{3}}{9}\right) \epsilon^{4}+\left(\frac{949 \pi^{6}}{15120}-\frac{196 \zeta_{3}^{2}}{9}\right) \epsilon^{5} \\
& +\mathcal{O}\left(\epsilon^{6}\right) \text {, } \\
& K_{S S}=-\frac{32}{\epsilon^{3}}+\frac{80 \pi^{2}}{3 \epsilon}+\frac{1984 \zeta_{3}}{3}+\frac{100 \pi^{4} \epsilon}{9}+\left(\frac{32704 \zeta_{5}}{5}-\frac{4960 \pi^{2} \zeta_{3}}{9}\right) \epsilon^{2} \\
& +\left(\frac{482 \pi^{6}}{63}-\frac{61504 \zeta_{3}^{2}}{9}\right) \epsilon^{3}+\mathcal{O}\left(\epsilon^{4}\right) \\
& K_{S A}=-\frac{44}{3 \epsilon^{2}}+\frac{1}{\epsilon}\left(\frac{4 \pi^{2}}{3}-\frac{268}{9}\right)+\left(56 \zeta_{3}+\frac{22 \pi^{2}}{3}-\frac{1616}{27}\right)+\left(\frac{2728 \zeta_{3}}{9}+\frac{88 \pi^{4}}{45}+\frac{134 \pi^{2}}{9}\right. \\
& \left.-\frac{9712}{81}\right) \epsilon+\left(\frac{16616 \zeta_{3}}{27}-\frac{716 \pi^{2} \zeta_{3}}{9}+296 \zeta_{5}+\frac{649 \pi^{4}}{90}+\frac{808 \pi^{2}}{27}-\frac{58304}{243}\right) \epsilon^{2} \\
& +\left(-\frac{3688 \zeta_{3}^{2}}{3}+\frac{100192 \zeta_{3}}{81}-\frac{1364 \pi^{2} \zeta_{3}}{9}+\frac{44968 \zeta_{5}}{15}-\frac{377 \pi^{6}}{1890}+\frac{3953 \pi^{4}}{270}+\frac{4856 \pi^{2}}{81}\right. \\
& \left.-\frac{349888}{729}\right) \epsilon^{3}+\mathcal{O}\left(\epsilon^{4}\right) \\
& K_{S f}=\frac{16}{3 \epsilon^{2}}+\frac{80}{9 \epsilon}+\left(\frac{448}{27}-\frac{8 \pi^{2}}{3}\right)+\left(-\frac{992 \zeta_{3}}{9}-\frac{40 \pi^{2}}{9}+\frac{2624}{81}\right) \epsilon \\
& +\left(-\frac{4960 \zeta_{3}}{27}-\frac{118 \pi^{4}}{45}-\frac{224 \pi^{2}}{27}+\frac{15616}{243}\right) \epsilon^{2} \\
& +\left(\frac{496 \pi^{2} \zeta_{3}}{9}-\frac{27776 \zeta_{3}}{81}-\frac{16352 \zeta_{5}}{15}-\frac{118 \pi^{4}}{27}-\frac{1312 \pi^{2}}{81}+\frac{93440}{729}\right) \epsilon^{3}+\mathcal{O}\left(\epsilon^{4}\right), \\
& K_{S S S}=-\frac{64}{\epsilon^{5}}+\frac{144 \pi^{2}}{\epsilon^{3}}+\frac{4544 \zeta_{3}}{\epsilon^{2}}+\frac{334 \pi^{4}}{5 \epsilon}+\left(\frac{497472 \zeta_{5}}{5}-10224 \pi^{2} \zeta_{3}\right) \\
& +\left(\frac{83933 \pi^{6}}{630}-161312 \zeta_{3}^{2}\right) \epsilon+\mathcal{O}\left(\epsilon^{2}\right)
\end{aligned}
$$




$$
\begin{aligned}
& K_{S S A}=-\frac{88}{\epsilon^{4}}+\frac{1}{\epsilon^{3}}\left(8 \pi^{2}-\frac{536}{3}\right)+\frac{1}{\epsilon^{2}}\left(336 \zeta_{3}+\frac{506 \pi^{2}}{3}-\frac{3232}{9}\right) \\
& +\frac{1}{\epsilon}\left(6248 \zeta_{3}+\frac{2 \pi^{4}}{5}+\frac{3082 \pi^{2}}{9}-\frac{19424}{27}\right) \\
& +\left(\frac{38056}{3} \zeta_{3}-1356 \pi^{2} \zeta_{3}+1776 \zeta_{5}+\frac{8767 \pi^{4}}{60}+\frac{18584 \pi^{2}}{27}-\frac{116608}{81}\right) \\
& +\left(-24288 \zeta_{3}^{2}+\frac{229472}{9} \zeta_{3}-\frac{35926}{3} \pi^{2} \zeta_{3}+\frac{684024 \zeta_{5}}{5}-\frac{4597 \pi^{6}}{140}+\frac{53399 \pi^{4}}{180}\right. \\
& \left.+\frac{111688 \pi^{2}}{81}-\frac{699776}{243}\right) \epsilon+\mathcal{O}\left(\epsilon^{2}\right), \\
& K_{S A A}=-\frac{968}{27 \epsilon^{3}}+\frac{1}{\epsilon^{2}}\left(\frac{176 \pi^{2}}{27}-\frac{13016}{81}\right)+\frac{1}{\epsilon}\left(\frac{3520 \zeta_{3}}{9}-\frac{88 \pi^{4}}{135}+\frac{4702 \pi^{2}}{81}-\frac{44372}{81}\right) \\
& +\left(\frac{10376}{3} \zeta_{3}-\frac{176}{9} \pi^{2} \zeta_{3}-384 \zeta_{5}+\frac{88 \pi^{4}}{5}+\frac{55202 \pi^{2}}{243}-\frac{1235050}{729}\right) \\
& +\left(-\frac{2216 \zeta_{3}^{2}}{3}+\frac{1089896}{81} \zeta_{3}-\frac{11968}{9} \pi^{2} \zeta_{3}+\frac{10912 \zeta_{5}}{3}-\frac{48701 \pi^{6}}{8505}+\frac{198197 \pi^{4}}{1620}\right. \\
& \left.+\frac{541345 \pi^{2}}{729}-\frac{10984045}{2187}\right) \epsilon+\mathcal{O}\left(\epsilon^{2}\right) \\
& K_{S S f}=\frac{32}{\epsilon^{4}}+\frac{160}{3 \epsilon^{3}}+\frac{1}{\epsilon^{2}}\left(\frac{896}{9}-\frac{184 \pi^{2}}{3}\right)+\frac{1}{\epsilon}\left(-2272 \zeta_{3}-\frac{920 \pi^{2}}{9}+\frac{5248}{27}\right) \\
& +\left(-\frac{11360 \zeta_{3}}{3}-\frac{797 \pi^{4}}{15}-\frac{5152 \pi^{2}}{27}+\frac{31232}{81}\right) \\
& +\left(\frac{13064 \pi^{2} \zeta_{3}}{3}-\frac{63616 \zeta_{3}}{9}-\frac{248736 \zeta_{5}}{5}-\frac{797 \pi^{4}}{9}-\frac{30176 \pi^{2}}{81}+\frac{186880}{243}\right) \epsilon \\
& +\mathcal{O}\left(\epsilon^{2}\right) \text {, } \\
& K_{S F f}=\frac{16}{3 \epsilon^{2}}+\frac{1}{\epsilon}\left(\frac{440}{9}-\frac{128 \zeta_{3}}{3}\right)+\left(-\frac{1216 \zeta_{3}}{9}-\frac{32 \pi^{4}}{45}-\frac{20 \pi^{2}}{3}+\frac{6844}{27}\right) \\
& +\left(\frac{160 \pi^{2} \zeta_{3}}{3}-\frac{21296 \zeta_{3}}{27}-\frac{896 \zeta_{5}}{3}-\frac{304 \pi^{4}}{135}-\frac{550 \pi^{2}}{9}+\frac{85454}{81}\right) \epsilon+\mathcal{O}\left(\epsilon^{2}\right), \\
& K_{S A f}=\frac{704}{27 \epsilon^{3}}+\frac{1}{\epsilon^{2}}\left(\frac{8528}{81}-\frac{64 \pi^{2}}{27}\right)+\frac{1}{\epsilon}\left(-\frac{896 \zeta_{3}}{9}-\frac{2960 \pi^{2}}{81}+\frac{26128}{81}\right)
\end{aligned}
$$




$$
\begin{aligned}
& +\left(-\frac{53600 \zeta_{3}}{27}-\frac{256 \pi^{4}}{45}-\frac{33628 \pi^{2}}{243}+\frac{656776}{729}\right) \\
& +\left(\frac{3872 \pi^{2} \zeta_{3}}{9}-\frac{22768 \zeta_{3}}{3}-1024 \zeta_{5}-\frac{5710 \pi^{4}}{81}-\frac{310628 \pi^{2}}{729}+\frac{5295988}{2187}\right) \epsilon \\
& +\mathcal{O}\left(\epsilon^{2}\right) \\
K_{S f f}= & -\frac{128}{27 \epsilon^{3}}-\frac{1280}{81 \epsilon^{2}}+\frac{1}{\epsilon}\left(\frac{160 \pi^{2}}{27}-\frac{128}{3}\right)+\left(\frac{8320 \zeta_{3}}{27}+\frac{1600 \pi^{2}}{81}-\frac{77824}{729}\right) \\
& +\left(\frac{83200 \zeta_{3}}{81}+\frac{868 \pi^{4}}{81}+\frac{160 \pi^{2}}{3}-\frac{560128}{2187}\right) \epsilon+\mathcal{O}\left(\epsilon^{2}\right) .
\end{aligned}
$$

\section{Definition of integral families}

In section 4, we defined one integral family for illustration. All eleven families share the same propagator denominators $\mathcal{D}_{1, \cdots, 6}$. Here we present the definition of the other propagator denominators $\mathcal{D}_{7, \cdots, 15}$ for the remaining ten families.

Family 2:

$$
\begin{aligned}
\mathcal{D}_{7} & =-n_{1} \cdot k_{1}, & \mathcal{D}_{8} & =-n_{1} \cdot k_{2}, & \mathcal{D}_{9} & =-n_{1} \cdot k_{3}, \\
\mathcal{D}_{10} & =-n_{2} \cdot\left(k_{3}-k_{1}\right), & \mathcal{D}_{11} & =-n_{2} \cdot\left(k_{3}-k_{2}\right), & & \mathcal{D}_{12}=-n_{2} \cdot k_{3}, \\
\mathcal{D}_{13} & =-n_{J} \cdot k_{1}-\omega, & \mathcal{D}_{14} & =-n_{J} \cdot k_{2}-\omega, & & \mathcal{D}_{15}=-n_{J} \cdot k_{3}-\omega .
\end{aligned}
$$

Family 3 :

$$
\begin{aligned}
& \mathcal{D}_{7}=-n_{1} \cdot k_{1}, \quad \mathcal{D}_{8}=-n_{1} \cdot k_{2}, \quad \mathcal{D}_{9}=-n_{1} \cdot\left(k_{3}-k_{2}\right), \\
& \mathcal{D}_{10}=-n_{2} \cdot k_{1}, \quad \mathcal{D}_{11}=-n_{2} \cdot k_{2}, \quad \mathcal{D}_{12}=-n_{2} \cdot\left(k_{3}-k_{2}\right) \text {, } \\
& \mathcal{D}_{13}=-n_{J} \cdot k_{1}-\omega, \quad \mathcal{D}_{14}=-n_{J} \cdot k_{2}-\omega, \quad \mathcal{D}_{15}=-n_{J} \cdot k_{3}-\omega .
\end{aligned}
$$

Family 4:

$$
\begin{aligned}
& \mathcal{D}_{7}=-n_{1} \cdot k_{1}, \quad \mathcal{D}_{8}=-n_{1} \cdot\left(k_{3}-k_{2}\right), \quad \mathcal{D}_{9}=-n_{1} \cdot k_{3}, \\
& \mathcal{D}_{10}=n_{2} \cdot k_{1}, \quad \mathcal{D}_{11}=-n_{2} \cdot\left(k_{2}-k_{1}\right), \quad \mathcal{D}_{12}=-n_{2} \cdot\left(k_{3}-k_{1}\right), \\
& \mathcal{D}_{13}=-n_{J} \cdot k_{3}-\omega, \quad \mathcal{D}_{14}=-n_{J} \cdot\left(k_{3}-k_{2}\right)-\omega, \quad \mathcal{D}_{15}=-n_{J} \cdot\left(k_{3}-k_{1}\right)-\omega .
\end{aligned}
$$

Family 5:

$$
\begin{aligned}
& \mathcal{D}_{7}=-n_{1} \cdot k_{1}, \quad \mathcal{D}_{8}=-n_{1} \cdot k_{2}, \quad \mathcal{D}_{9}=-n_{1} \cdot k_{3}, \\
& \mathcal{D}_{10}=-n_{2} \cdot\left(k_{1}-k_{2}\right), \quad \mathcal{D}_{11}=-n_{2} \cdot\left(k_{3}-k_{2}\right), \quad \mathcal{D}_{12}=-n_{2} \cdot k_{3}, \\
& \mathcal{D}_{13}=-n_{J} \cdot k_{1}-\omega, \quad \mathcal{D}_{14}=-n_{J} \cdot k_{2}-\omega, \quad \mathcal{D}_{15}=-n_{J} \cdot k_{3}-\omega .
\end{aligned}
$$

Family 6:

$$
\begin{aligned}
& \mathcal{D}_{7}=-n_{1} \cdot k_{1}, \quad \mathcal{D}_{8}=-n_{1} \cdot\left(k_{1}-k_{2}\right), \quad \mathcal{D}_{9}=-n_{1} \cdot\left(k_{3}-k_{2}\right), \\
& \mathcal{D}_{10}=-n_{2} \cdot k_{2}, \quad \mathcal{D}_{11}=-n_{2} \cdot k_{3}, \quad \mathcal{D}_{12}=-n_{2} \cdot\left(k_{3}-k_{1}\right) \text {, } \\
& \mathcal{D}_{13}=-n_{J} \cdot k_{1}-\omega, \quad \mathcal{D}_{14}=-n_{J} \cdot k_{2}-\omega, \quad \mathcal{D}_{15}=-n_{J} \cdot k_{3}-\omega .
\end{aligned}
$$




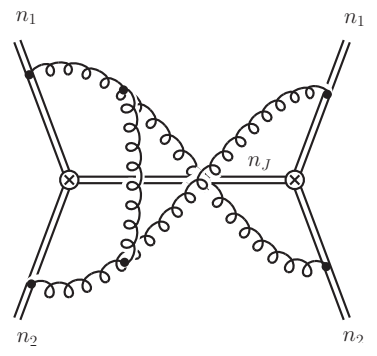

Figure 5. Three-loop Feynman diagram with imaginary part for $n_{12}>0$.

Family 7:

$$
\begin{aligned}
& \mathcal{D}_{7}=-n_{1} \cdot k_{1}, \quad \mathcal{D}_{8}=-n_{1} \cdot\left(k_{1}-k_{3}\right), \quad \mathcal{D}_{9}=-n_{1} \cdot\left(k_{2}-k_{3}\right), \\
& \mathcal{D}_{10}=-n_{2} \cdot k_{1}, \quad \mathcal{D}_{11}=-n_{2} \cdot\left(k_{1}-k_{2}\right), \quad \mathcal{D}_{12}=-n_{2} \cdot\left(k_{1}-k_{3}\right), \\
& \mathcal{D}_{13}=-n_{J} \cdot k_{1}-\omega, \quad \mathcal{D}_{14}=-n_{J} \cdot\left(k_{1}-k_{3}\right)-\omega, \quad \mathcal{D}_{15}=-n_{J} \cdot\left(k_{2}-k_{3}\right)-\omega .
\end{aligned}
$$

Family 8:

$$
\begin{aligned}
& \mathcal{D}_{7}=-n_{1} \cdot k_{1}, \quad \mathcal{D}_{8}=-n_{1} \cdot k_{2}, \quad \mathcal{D}_{9}=-n_{1} \cdot k_{3}, \\
& \mathcal{D}_{10}=-n_{2} \cdot\left(k_{2}-k_{1}\right), \quad \mathcal{D}_{11}=-n_{2} \cdot\left(k_{3}-k_{1}\right), \quad \mathcal{D}_{12}=-n_{2} \cdot k_{3}, \\
& \mathcal{D}_{13}=-n_{J} \cdot\left(k_{3}-k_{1}\right)-\omega, \quad \mathcal{D}_{14}=-n_{J} \cdot k_{2}-\omega, \quad \mathcal{D}_{15}=-n_{J} \cdot k_{3}-\omega .
\end{aligned}
$$

Family 9:

$$
\begin{aligned}
& \mathcal{D}_{7}=-n_{1} \cdot k_{1}, \quad \mathcal{D}_{8}=-n_{1} \cdot\left(k_{3}-k_{1}\right), \quad \mathcal{D}_{9}=-n_{1} \cdot\left(k_{3}-k_{2}\right), \\
& \mathcal{D}_{10}=-n_{2} \cdot k_{1}, \quad \mathcal{D}_{11}=-n_{2} \cdot\left(k_{3}-k_{1}\right), \quad \mathcal{D}_{12}=-n_{2} \cdot\left(k_{2}-k_{1}\right), \\
& \mathcal{D}_{13}=-n_{J} \cdot k_{3}-\omega, \quad \mathcal{D}_{14}=-n_{J} \cdot\left(k_{3}-k_{1}\right)-\omega, \quad \mathcal{D}_{15}=-n_{J} \cdot\left(k_{3}-k_{2}\right)-\omega .
\end{aligned}
$$

Family 10:

$$
\begin{aligned}
& \mathcal{D}_{7}=-n_{1} \cdot\left(k_{1}-k_{2}\right), \quad \mathcal{D}_{8}=-n_{1} \cdot\left(k_{3}-k_{2}\right), \quad \mathcal{D}_{9}=-n_{1} \cdot k_{3}, \\
& \mathcal{D}_{10}=-n_{2} \cdot k_{1}, \quad \mathcal{D}_{11}=-n_{2} \cdot\left(k_{1}-k_{2}\right), \quad \mathcal{D}_{12}=-n_{2} \cdot k_{3}, \\
& \mathcal{D}_{13}=-n_{J} \cdot k_{1}-\omega, \quad \mathcal{D}_{14}=-n_{J} \cdot\left(k_{1}-k_{2}\right)-\omega, \quad \mathcal{D}_{15}=-n_{J} \cdot k_{3}-\omega .
\end{aligned}
$$

Family 11:

$$
\begin{aligned}
& \mathcal{D}_{7}=-n_{1} \cdot\left(k_{1}-k_{2}\right), \quad \mathcal{D}_{8}=-n_{1} \cdot k_{2}, \quad \mathcal{D}_{9}=-n_{1} \cdot\left(k_{1}-k_{3}\right), \\
& \mathcal{D}_{10}=-n_{2} \cdot\left(k_{1}-k_{2}\right), \quad \mathcal{D}_{11}=-n_{2} \cdot\left(k_{3}-k_{2}\right), \quad \mathcal{D}_{12}=-n_{2} \cdot k_{3}, \\
& \mathcal{D}_{13}=-n_{J} \cdot k_{1}-\omega, \quad \mathcal{D}_{14}=-n_{J} \cdot k_{2}-\omega, \quad \mathcal{D}_{15}=-n_{J} \cdot\left(k_{1}-k_{2}+k_{3}\right)-\omega \text {. }
\end{aligned}
$$

\section{Feynman parameter integrations with HyperInt}

Here we demonstrate the calculation of the three-loop MIs with an imaginary part related to a physical threshold (similar to the two-loop example in section 3) using HyperInt [62]. 
As a concrete example we consider one of the most complicated MIs of this kind, namely the integral $G_{1,1,0,1,1,1,1,0,1,1,0,1,0,0,1}^{[6]}$ in family $6,{ }^{15}$ which contributes to the Feynman diagram in figure 5 . The corresponding $\mathcal{U}$ and $\mathcal{F}$ polynomials in the Feynman parameter $\left(x_{i}\right)$ representation, see e.g. ref. [85], are given by

$$
\begin{aligned}
\mathcal{U}= & x_{1} x_{2} x_{5}+x_{1} x_{4} x_{5}+x_{2} x_{4} x_{5}+x_{1} x_{6} x_{5}+x_{2} x_{6} x_{5}+x_{1} x_{2} x_{6}+x_{1} x_{4} x_{6}+x_{2} x_{4} x_{6}, \\
\mathcal{F}= & \frac{n_{12}}{2} x_{4} x_{5} x_{7} x_{10}+\frac{n_{12}}{2} x_{4} x_{6} x_{7} x_{10}+\frac{n_{12}}{2} x_{5} x_{6} x_{7} x_{10}-\frac{n_{12}}{2} x_{1} x_{6} x_{9} x_{10}-\frac{n_{12}}{2} x_{2} x_{5} x_{7} x_{12} \\
& +\frac{n_{12}}{2} x_{1} x_{2} x_{9} x_{12}+\frac{n_{12}}{2} x_{1} x_{4} x_{9} x_{12}+\frac{n_{12}}{2} x_{2} x_{4} x_{9} x_{12}+x_{1} x_{5} x_{10} x_{15}+x_{4} x_{5} x_{15} x_{10} \\
& +x_{5} x_{6} x_{10} x_{15}+x_{1} x_{2} x_{5} x_{15}+x_{1} x_{4} x_{5} x_{15}+x_{2} x_{4} x_{5} x_{15}+x_{1} x_{2} x_{6} x_{15}+x_{1} x_{4} x_{6} x_{15} \\
& +x_{2} x_{4} x_{6} x_{15}+x_{1} x_{5} x_{6} x_{15}+x_{2} x_{5} x_{6} x_{15}+x_{4} x_{5} x_{7} x_{15}+x_{2} x_{6} x_{7} x_{15}+x_{4} x_{6} x_{7} x_{15} \\
& +x_{5} x_{6} x_{7} x_{15}+x_{1} x_{2} x_{9} x_{15}+x_{1} x_{4} x_{9} x_{15}+x_{2} x_{4} x_{9} x_{15}+x_{2} x_{6} x_{9} x_{15}+x_{1} x_{2} x_{12} x_{15} \\
& +x_{1} x_{4} x_{12} x_{15}+x_{2} x_{4} x_{12} x_{15}+x_{1} x_{5} x_{12} x_{15}+x_{4} x_{6} x_{10} x_{15}
\end{aligned}
$$

where we set $n_{1}^{2}=n_{2}^{2}=n_{J}^{2}=0, n_{1 J}=n_{2 J}=2, \omega=-1$ for convenience. The dependence on these variables can be easily restored according to eq. (4.4). The index $i$ of the $x_{i}$ refers to the corresponding propagator in family 6 . Note that our definition of the integrals in eq. (4.2) implies that an infinitesimal $-\mathrm{i} 0$ is to be added to $\mathcal{F}$ in eq. (D.2).

By a dimensional recurrence relation, $G_{1,1,0,1,1,1,1,0,1,1,0,1,0,0,1}^{[6]}$ can be related to the integral $G_{2,1,0,2,2,1,1,0,1,1,0,2,0,0,4}^{[6]}$, which is finite in $d=8-2 \epsilon$ dimensions, and less complicated already known integrals. The coefficients of the integrals in such relations are real and only depend on $d$. Regardless of the sign of $n_{12}$, the $\mathcal{F}$ polynomial becomes negative in certain integration regions. We now exploit the projective nature of the Feynman parameter representation [85] by fixing $x_{4}=1$ and are left with the integrations over the remaining $x_{i}$ from 0 to $+\infty$. For $n_{12}=2$ and dropping the infinitesimal $-\mathrm{i} 0$ HyperInt manages to perform the $x_{7}, x_{9}, x_{15}, x_{10}, x_{12}, x_{1}, x_{2}, x_{5}, x_{6}$ integrations in that order. Because we have removed the infinitesimal -i0, HyperInt automatically adds an imaginary infinitesimal $\delta_{x_{6}} \mathrm{i} \varepsilon$ with $\delta_{x_{6}}= \pm 1$ to the parameter $x_{6}$ in order to deform the integration contour away from an (integrable) threshold singularity for $\mathcal{F} \rightarrow 0$. Note that, in contrast to the original integral with fixed $-\mathrm{i} 0$, the sign of the imaginary infinitesimal now depends on the integration region. The HyperInt output will therefore in general differ from the integral we aim to compute. This difference however only affects the imaginary part of the integral, which is irrelevant for our purpose as discussed in section 4 . The real part agrees with the original

\footnotetext{
${ }^{15}$ Here we use the notation $G_{a_{1}, \ldots, e_{3}}^{[6]} \equiv G\left(a_{1}, \ldots, e_{3}\right)$ for the integrals in family 6 defined according to eq. (4.2).
} 
one. To see this we write

$$
\begin{aligned}
\operatorname{Re}[G(\vec{a}, \vec{b}, \vec{c}, \vec{e}, \epsilon)]= & \frac{1}{2}\left(\left.G(\vec{a}, \vec{b}, \vec{c}, \vec{e}, \epsilon)\right|_{+\mathrm{i} 0}+\left.G(\vec{a}, \vec{b}, \vec{c}, \vec{e}, \epsilon)\right|_{-\mathrm{i} 0}\right) \\
= & \frac{1}{2}\left(\left.G(\vec{a}, \vec{b}, \vec{c}, \vec{e}, \epsilon)\right|_{+\mathrm{i} 0} ^{>}+\left.G(\vec{a}, \vec{b}, \vec{c}, \vec{e}, \epsilon)\right|_{+\mathrm{i} 0} ^{<}\right. \\
& \left.+\left.G(\vec{a}, \vec{b}, \vec{c}, \vec{e}, \epsilon)\right|_{-\mathrm{i} 0} ^{>}+\left.G(\vec{a}, \vec{b}, \vec{c}, \vec{e}, \epsilon)\right|_{-\mathrm{i} 0} ^{<}\right) \\
= & \frac{1}{2}\left(\left.G(\vec{a}, \vec{b}, \vec{c}, \vec{e}, \epsilon)\right|_{+\delta_{x_{k}} \mathrm{i} \varepsilon} ^{>}+\left.G(\vec{a}, \vec{b}, \vec{c}, \vec{e}, \epsilon)\right|_{-\delta_{x_{k}} \mathrm{i} \varepsilon} ^{<}\right. \\
& \left.+\left.G(\vec{a}, \vec{b}, \vec{c}, \vec{e}, \epsilon)\right|_{-\delta_{x_{k}} \mathrm{i} \varepsilon} ^{>}+\left.G(\vec{a}, \vec{b}, \vec{c}, \vec{e}, \epsilon)\right|_{+\delta_{x_{k}} \mathrm{i} \varepsilon} ^{<}\right) \\
= & \operatorname{Re}\left[\left.G(\vec{a}, \vec{b}, \vec{c}, \vec{e}, \epsilon)\right|_{+\delta_{x_{k}} \mathrm{i} \varepsilon} ^{>}+\left.G(\vec{a}, \vec{b}, \vec{c}, \vec{e}, \epsilon)\right|_{+\delta_{x_{k}} \mathrm{i} \varepsilon} ^{<}\right] \\
= & \operatorname{Re}\left[\left.G(\vec{a}, \vec{b}, \vec{c}, \vec{e}, \epsilon)\right|_{+\delta_{x_{k}} \mathrm{i} \varepsilon}\right]
\end{aligned}
$$

for a generic integral $G{ }^{16}$ The subscript indicates whether the integral is evaluated with fixed $\pm \mathrm{i} 0$ or an imaginary infinitesimal $\delta_{x_{k}} \mathrm{i} \varepsilon$ added to the Feynman parameter $x_{k}$ in the $\mathcal{F}$ polynomial. The $>(<)$ superscript indicates the contribution from all integration regions, where the coefficient of $x_{k}$ (and therefore of $\delta_{x_{k}} \mathrm{i} \varepsilon$ ) is positive (negative). We have checked eq. (D.3) by computing our quasi-finite integrals for different $x_{i}$ initially fixed to one and different integration orders, which causes HyperInt to assign the $\delta_{x_{k}}$ i $\varepsilon$ to different $x_{k}$. In most cases we found a configuration, where HyperInt adds the imaginary infinitesimal to a Feynman parameter with positive definite coefficient, which is equivalent to a fixed $-\mathrm{i} 0$ in the $\mathcal{F}$ polynomial. In this way we explicitly confirmed that it is irrelevant for the real part of the result whether the coefficient of the $\delta_{x_{k}}$ i $\varepsilon$ is positive or varies in sign depending on the integration region.

With the output from HyperInt we obtain for our concrete example in $d=8-2 \epsilon$ dimensions $\left(\delta_{x_{6}}^{2}=1\right)$,

$$
\begin{aligned}
& \operatorname{Re}\left[G_{2,1,0,2,2,1,1,0,1,1,0,2,0,0,4]}^{[6]}=\left(\frac{1}{72}+\frac{7 \pi^{2}}{72}\right) \zeta_{3}-\frac{25 \zeta_{5}}{72}-\frac{29 \pi^{4}}{8640}-\frac{11 \pi^{2}}{432}+\frac{\pi^{2}}{48} \delta_{x_{6}}^{2}\right. \\
& +\left[\frac{\pi^{2}}{18} \zeta_{1,-3}+\frac{17 \zeta_{3}^{2}}{288}-\frac{611 \pi^{2} \zeta_{3}}{1728}+\frac{221 \zeta_{3}}{216}-\frac{1693 \zeta_{5}}{1728}+\frac{2021 \pi^{6}}{544320}-\frac{\pi^{4}}{64}-\frac{823 \pi^{2}}{5184}\right. \\
& \left.+\frac{7 \pi^{2}}{24} \ln 2+\left(\frac{3 \pi^{2} \zeta_{3}}{16}-\frac{\pi^{6}}{540}+\frac{\pi^{4}}{288}+\frac{17 \pi^{2}}{192}\right) \delta_{x_{6}}^{2}\right] \epsilon \\
& +\left[\frac{7 \zeta_{3}}{36} \zeta_{1,-3}-\frac{257 \pi^{2}}{432} \zeta_{1,-3}-\frac{7}{12} \zeta_{1,-3}+\frac{28}{51} \zeta_{1,1,-5}-\frac{\pi^{2}}{18} \zeta_{1,1,-3}-\frac{14}{153} \zeta_{1,3,-3}\right. \\
& -\frac{10417 \zeta_{3}^{2}}{6912}+\frac{29215 \pi^{4} \zeta_{3}}{88128}-\frac{1937 \pi^{2} \zeta_{3}}{2592}+\frac{5627 \zeta_{3}}{1296}+\frac{30181 \pi^{2} \zeta_{5}}{58752} \\
& -\frac{2111 \zeta_{5}}{1296}-\frac{335531 \zeta_{7}}{39168}-\frac{53731 \pi^{6}}{1306368}+\frac{3869 \pi^{4}}{25920}-\frac{14965 \pi^{2}}{31104} \\
& { }^{16} \text { On the other hand we have } \operatorname{Im}\left[\left.G(\vec{a}, \vec{b}, \vec{c}, \vec{e}, \epsilon)\right|_{-\mathrm{i} 0}\right]=\operatorname{Im}\left[\left.G(\vec{a}, \vec{b}, \vec{c}, \vec{e}, \epsilon)\right|_{-\delta_{x_{k}} \mathrm{i} \varepsilon} ^{>}\right]- \\
& \operatorname{Im}\left[+\left.G(\vec{a}, \vec{b}, \vec{c}, \vec{e}, \epsilon)\right|_{-\delta_{x_{k}} \mathrm{i \varepsilon}} ^{<}\right] .
\end{aligned}
$$




$$
\begin{aligned}
& +\left(\frac{49 \zeta_{3}}{48}+\frac{71 \pi^{2}}{48}\right) \ln 2+\frac{7 \pi^{2}}{48} \ln ^{2} 2+\left(-\frac{\pi^{4}}{4} \zeta_{3}+\frac{\pi^{6}}{48}-\frac{\pi^{4}}{24}\right) \delta_{x_{6}}^{4} \\
& \left.+\left(\frac{29 \pi^{4} \zeta_{3}}{96}+\frac{9 \pi^{2} \zeta_{3}}{16}+\frac{7 \pi^{2} \zeta_{5}}{32}-\frac{113 \pi^{6}}{103680}-\frac{187 \pi^{4}}{864}+\frac{355 \pi^{2}}{1152}\right) \delta_{x_{6}}^{2}\right] \epsilon^{2} \\
& +\mathcal{O}\left(\epsilon^{3}\right),
\end{aligned}
$$

where we give all terms in the $\epsilon$ expansion required for our soft function calculation. Note that the multiple zeta values, the $\ln 2$ terms, and the terms of transcendental weight $>6$ cancel among the different MIs and do not appear in the final result for the soft function.

Open Access. This article is distributed under the terms of the Creative Commons Attribution License (CC-BY 4.0), which permits any use, distribution and reproduction in any medium, provided the original author(s) and source are credited.

\section{References}

[1] R. Boughezal, F. Caola, K. Melnikov, F. Petriello and M. Schulze, Higgs boson production in association with a jet at next-to-next-to-leading order in perturbative QCD, JHEP 06 (2013) 072 [arXiv: 1302.6216] [INSPIRE].

[2] X. Chen, T. Gehrmann, E.W.N. Glover and M. Jaquier, Precise QCD predictions for the production of Higgs + jet final states, Phys. Lett. B $\mathbf{7 4 0}$ (2015) 147 [arXiv:1408.5325] [INSPIRE].

[3] R. Boughezal, C. Focke, X. Liu and F. Petriello, $W$-boson production in association with a jet at next-to-next-to-leading order in perturbative QCD, Phys. Rev. Lett. 115 (2015) 062002 [arXiv: 1504.02131] [INSPIRE].

[4] R. Boughezal, F. Caola, K. Melnikov, F. Petriello and M. Schulze, Higgs boson production in association with a jet at next-to-next-to-leading order, Phys. Rev. Lett. 115 (2015) 082003 [arXiv: 1504.07922] [INSPIRE].

[5] R. Boughezal, C. Focke, W. Giele, X. Liu and F. Petriello, Higgs boson production in association with a jet at NNLO using jettiness subtraction, Phys. Lett. B $\mathbf{7 4 8}$ (2015) 5 [arXiv: 1505.03893] [INSPIRE].

[6] F. Caola, K. Melnikov and M. Schulze, Fiducial cross sections for Higgs boson production in association with a jet at next-to-next-to-leading order in QCD, Phys. Rev. D 92 (2015) 074032 [arXiv: 1508.02684] [INSPIRE].

[7] A. Gehrmann-De Ridder, T. Gehrmann, E.W.N. Glover, A. Huss and T.A. Morgan, Precise $Q C D$ predictions for the production of a $Z$ boson in association with a hadronic jet, Phys. Rev. Lett. 117 (2016) 022001 [arXiv:1507.02850] [INSPIRE].

[8] R. Boughezal et al., Z-boson production in association with a jet at next-to-next-to-leading order in perturbative QCD, Phys. Rev. Lett. 116 (2016) 152001 [arXiv:1512.01291] [INSPIRE].

[9] A. Gehrmann-De Ridder, T. Gehrmann, E.W.N. Glover, A. Huss and T.A. Morgan, The NNLO QCD corrections to Z boson production at large transverse momentum, JHEP 07 (2016) 133 [arXiv: 1605.04295] [INSPIRE]. 
[10] J.M. Campbell, R.K. Ellis and C. Williams, Direct photon production at next-to-next-to-leading order, Phys. Rev. Lett. 118 (2017) 222001 [Erratum ibid. 124 (2020) 259901] [arXiv: 1612.04333] [INSPIRE].

[11] X. Chen, J. Cruz-Martinez, T. Gehrmann, E.W.N. Glover and M. Jaquier, NNLO QCD corrections to Higgs boson production at large transverse momentum, JHEP 10 (2016) 066 [arXiv: 1607.08817] [INSPIRE].

[12] J.M. Campbell, R.K. Ellis and C. Williams, Driving missing data at the LHC: NNLO predictions for the ratio of $\gamma+j$ and $Z+j$, Phys. Rev. D 96 (2017) 014037 [arXiv: 1703.10109] [INSPIRE].

[13] A. Gehrmann-De Ridder, T. Gehrmann, E.W.N. Glover, A. Huss and D.M. Walker, Next-to-next-to-leading-order QCD corrections to the transverse momentum distribution of weak gauge bosons, Phys. Rev. Lett. 120 (2018) 122001 [arXiv:1712.07543] [INSPIRE].

[14] X. Chen, T. Gehrmann, N. Glover, M. Höfer and A. Huss, Isolated photon and photon+jet production at NNLO QCD accuracy, JHEP 04 (2020) 166 [arXiv:1904.01044] [INSPIRE].

[15] X. Chen, T. Gehrmann, E.W.N. Glover and A. Huss, Fiducial cross sections for the four-lepton decay mode in Higgs-plus-jet production up to NNLO QCD, JHEP 07 (2019) 052 [arXiv: 1905.13738] [INSPIRE].

[16] J.M. Campbell, R.K. Ellis and S. Seth, $H+1$ jet production revisited, JHEP 10 (2019) 136 [arXiv: 1906.01020] [INSPIRE].

[17] E. Laenen, G. Oderda and G.F. Sterman, Resummation of threshold corrections for single particle inclusive cross-sections, Phys. Lett. B 438 (1998) 173 [hep-ph/9806467] [InSPIRE].

[18] T. Becher and M.D. Schwartz, Direct photon production with effective field theory, JHEP 02 (2010) 040 [arXiv: 0911.0681] [INSPIRE].

[19] C.W. Bauer, S. Fleming and M.E. Luke, Summing Sudakov logarithms in $B \rightarrow X_{s} \gamma$ in effective field theory, Phys. Rev. D 63 (2000) 014006 [hep-ph/0005275] [INSPIRE].

[20] C.W. Bauer, S. Fleming, D. Pirjol and I.W. Stewart, An effective field theory for collinear and soft gluons: heavy to light decays, Phys. Rev. D 63 (2001) 114020 [hep-ph/0011336] [INSPIRE].

[21] C.W. Bauer and I.W. Stewart, Invariant operators in collinear effective theory, Phys. Lett. B 516 (2001) 134 [hep-ph/0107001] [INSPIRE].

[22] C.W. Bauer, D. Pirjol and I.W. Stewart, Soft collinear factorization in effective field theory, Phys. Rev. D 65 (2002) 054022 [hep-ph/0109045] [INSPIRE].

[23] C.W. Bauer, S. Fleming, D. Pirjol, I.Z. Rothstein and I.W. Stewart, Hard scattering factorization from effective field theory, Phys. Rev. D 66 (2002) 014017 [hep-ph/0202088] [INSPIRE].

[24] M. Beneke, A.P. Chapovsky, M. Diehl and T. Feldmann, Soft collinear effective theory and heavy to light currents beyond leading power, Nucl. Phys. B 643 (2002) 431 [hep-ph/0206152] [INSPIRE].

[25] T. Becher, C. Lorentzen and M.D. Schwartz, Resummation for $W$ and $Z$ production at large $p_{T}$, Phys. Rev. Lett. 108 (2012) 012001 [arXiv:1106.4310] [inSPIRE].

[26] T. Becher, C. Lorentzen and M.D. Schwartz, Precision direct photon and W-boson spectra at high $p_{T}$ and comparison to LHC data, Phys. Rev. D 86 (2012) 054026 [arXiv:1206.6115] [INSPIRE].

[27] T. Becher, G. Bell, C. Lorentzen and S. Marti, Transverse-momentum spectra of electroweak bosons near threshold at NNLO, JHEP 02 (2014) 004 [arXiv: 1309.3245] [INSPIRE]. 
[28] F.P. Huang, C.S. Li, H.T. Li and J. Wang, Renormalization-group improved predictions for Higgs boson production at large $p_{T}$, Phys. Rev. D 90 (2014) 094024 [arXiv:1406.2591] [INSPIRE].

[29] T. Becher, G. Bell, C. Lorentzen and S. Marti, The transverse-momentum spectrum of Higgs bosons near threshold at NNLO, JHEP 11 (2014) 026 [arXiv:1407.4111] [INSPIRE].

[30] M.D. Schwartz, Precision direct photon spectra at high energy and comparison to the $8 \mathrm{TeV}$ ATLAS data, JHEP 09 (2016) 005 [arXiv: 1606.02313] [INSPIRE].

[31] L.G. Almeida, S.D. Ellis, C. Lee, G. Sterman, I. Sung and J.R. Walsh, Comparing and counting logs in direct and effective methods of QCD resummation, JHEP 04 (2014) 174 [arXiv: 1401.4460] [INSPIRE].

[32] Q. Jin and H. Lüo, Analytic form of the three-loop four-gluon scattering amplitudes in Yang-Mills theory, arXiv:1910.05889 [INSPIRE].

[33] T. Ahmed, J. Henn and B. Mistlberger, Four-particle scattering amplitudes in QCD at NNLO to higher orders in the dimensional regulator, JHEP 12 (2019) 177 [arXiv: 1910.06684] [INSPIRE].

[34] R. Brüser, Z.L. Liu and M. Stahlhofen, Three-loop quark jet function, Phys. Rev. Lett. 121 (2018) 072003 [arXiv: 1804.09722] [INSPIRE].

[35] P. Banerjee, P.K. Dhani and V. Ravindran, Gluon jet function at three loops in QCD, Phys. Rev. D 98 (2018) 094016 [arXiv: 1805. 02637] [InSPIRE].

[36] L.J. Dixon, E. Herrmann, K. Yan and H.X. Zhu, Soft gluon emission at two loops in full color, JHEP 05 (2020) 135 [arXiv: 1912.09370] [INSPIRE].

[37] Y.J. Zhu, Double soft current at one-loop in QCD, arXiv:2009.08919 [INSPIRE].

[38] S. Catani, D. Colferai and A. Torrini, Triple (and quadruple) soft-gluon radiation in $Q C D$ hard scattering, JHEP 01 (2020) 118 [arXiv:1908.01616] [INSPIRE].

[39] R. Brüser, Z.L. Liu and M. Stahlhofen, Three-loop soft function for heavy-to-light quark decays, JHEP 03 (2020) 071 [arXiv: 1911.04494] [INSPIRE].

[40] L.W. Garland, T. Gehrmann, E.W.N. Glover, A. Koukoutsakis and E. Remiddi, Two loop QCD helicity amplitudes for $e^{+} e^{-} \rightarrow$ three jets, Nucl. Phys. B 642 (2002) 227 [hep-ph/0206067] [INSPIRE].

[41] T. Gehrmann and L. Tancredi, Two-loop QCD helicity amplitudes for $q \bar{q} \rightarrow W^{ \pm} \gamma$ and $q \bar{q} \rightarrow Z^{0} \gamma$, JHEP 02 (2012) 004 [arXiv:1112.1531] [INSPIRE].

[42] Q. Jin and G. Yang, Analytic two-loop Higgs amplitudes in effective field theory and the maximal transcendentality principle, Phys. Rev. Lett. 121 (2018) 101603 [arXiv: 1804.04653] [INSPIRE].

[43] Q. Jin and G. Yang, Hidden analytic relations for two-loop Higgs amplitudes in QCD, Commun. Theor. Phys. 72 (2020) 065201 [arXiv: 1904.07260] [INSPIRE].

[44] T. Becher, G. Bell and S. Marti, NNLO soft function for electroweak boson production at large transverse momentum, JHEP 04 (2012) 034 [arXiv: 1201.5572] [INSPIRE].

[45] I.W. Stewart, F.J. Tackmann and W.J. Waalewijn, The quark beam function at NNLL, JHEP 09 (2010) 005 [arXiv: 1002.2213] [inSPIRE].

[46] S. Catani and M.H. Seymour, The dipole formalism for the calculation of QCD jet cross-sections at next-to-leading order, Phys. Lett. B 378 (1996) 287 [hep-ph/9602277] [INSPIRE]. 
[47] S. Catani and M.H. Seymour, A general algorithm for calculating jet cross-sections in NLO QCD, Nucl. Phys. B 485 (1997) 291 [Erratum ibid. 510 (1998) 503] [hep-ph/9605323] [INSPIRE].

[48] I. Moult, I.W. Stewart, F.J. Tackmann and W.J. Waalewijn, Employing helicity amplitudes for resummation, Phys. Rev. D 93 (2016) 094003 [arXiv: 1508. 02397] [INSPIRE].

[49] C.M. Arnesen, J. Kundu and I.W. Stewart, Constraint equations for heavy-to-light currents in SCET, Phys. Rev. D 72 (2005) 114002 [hep-ph/0508214] [INSPIRE].

[50] P. Nogueira, Automatic Feynman graph generation, J. Comput. Phys. 105 (1993) 279 [INSPIRE].

[51] R. Brüser, Renormalization of Wilson lines and applications to scattering processes, Ph.D. thesis, University of Mainz, Mainz, Germany (2019).

[52] A. Pak, The toolbox of modern multi-loop calculations: novel analytic and semi-analytic techniques, J. Phys. Conf. Ser. 368 (2012) 012049 [arXiv:1111.0868] [InSPIRE].

[53] A.V. Smirnov, FIRE5: a C++ implementation of Feynman Integral REduction, Comput. Phys. Commun. 189 (2015) 182 [arXiv:1408.2372] [INSPIRE].

[54] E. Panzer, On hyperlogarithms and Feynman integrals with divergences and many scales, JHEP 03 (2014) 071 [arXiv: 1401.4361] [INSPIRE].

[55] A. von Manteuffel, E. Panzer and R.M. Schabinger, A quasi-finite basis for multi-loop Feynman integrals, JHEP 02 (2015) 120 [arXiv:1411.7392] [INSPIRE].

[56] A. von Manteuffel, E. Panzer and R.M. Schabinger, On the computation of form factors in massless QCD with finite master integrals, Phys. Rev. D 93 (2016) 125014 [arXiv: 1510.06758] [INSPIRE].

[57] Z.L. Liu and M. Neubert, Two-loop radiative jet function for exclusive B-meson and Higgs decays, JHEP 06 (2020) 060 [arXiv:2003.03393] [INSPIRE].

[58] O.V. Tarasov, Connection between Feynman integrals having different values of the space-time dimension, Phys. Rev. D 54 (1996) 6479 [hep-th/9606018] [INSPIRE].

[59] R.N. Lee, Space-time dimensionality $D$ as complex variable: calculating loop integrals using dimensional recurrence relation and analytical properties with respect to D, Nucl. Phys. B 830 (2010) 474 [arXiv: 0911.0252] [INSPIRE].

[60] R.N. Lee, Calculating multiloop integrals using dimensional recurrence relation and D-analyticity, Nucl. Phys. B Proc. Suppl. 205-206 (2010) 135 [arXiv:1007.2256] [InSPIRE].

[61] A. von Manteuffel and C. Studerus, Reduze 2 - distributed Feynman integral reduction, arXiv:1201.4330 [INSPIRE].

[62] E. Panzer, Algorithms for the symbolic integration of hyperlogarithms with applications to Feynman integrals, Comput. Phys. Commun. 188 (2015) 148 [arXiv:1403.3385] [INSPIRE].

[63] R.N. Lee, Presenting LiteRed: a tool for the Loop InTEgrals REDuction, arXiv:1212.2685 [INSPIRE].

[64] R.N. Lee, LiteRed 1.4: a powerful tool for reduction of multiloop integrals, J. Phys. Conf. Ser. 523 (2014) 012059 [arXiv: 1310.1145] [INSPIRE].

[65] A.V. Smirnov, FIESTA4: optimized Feynman integral calculations with GPU support, Comput. Phys. Commun. 204 (2016) 189 [arXiv:1511.03614] [INSPIRE].

[66] E. Gardi, E. Laenen, G. Stavenga and C.D. White, Webs in multiparton scattering using the replica trick, JHEP 11 (2010) 155 [arXiv:1008.0098] [INSPIRE]. 
[67] E. Gardi, J.M. Smillie and C.D. White, The non-Abelian exponentiation theorem for multiple Wilson lines, JHEP 06 (2013) 088 [arXiv: 1304.7040] [INSPIRE].

[68] T. Becher, M. Neubert and B.D. Pecjak, Factorization and momentum-space resummation in deep-inelastic scattering, JHEP 01 (2007) 076 [hep-ph/0607228] [INSPIRE].

[69] T. Becher and M. Neubert, On the structure of infrared singularities of gauge-theory amplitudes, JHEP 06 (2009) 081 [Erratum ibid. 11 (2013) 024] [arXiv: 0903.1126] [INSPIRE].

[70] T. Becher and M. Neubert, Infrared singularities of scattering amplitudes and $N^{3} L L$ resummation for $n$-jet processes, JHEP 01 (2020) 025 [arXiv: 1908.11379] [INSPIRE].

[71] O. Almelid, C. Duhr and E. Gardi, Three-loop corrections to the soft anomalous dimension in multileg scattering, Phys. Rev. Lett. 117 (2016) 172002 [arXiv:1507.00047] [INSPIRE].

[72] T. van Ritbergen, A.N. Schellekens and J.A.M. Vermaseren, Group theory factors for Feynman diagrams, Int. J. Mod. Phys. A 14 (1999) 41 [hep-ph/9802376] [INSPIRE].

[73] R.N. Lee, A.V. Smirnov, V.A. Smirnov and M. Steinhauser, Four-loop quark form factor with quartic fundamental colour factor, JHEP 02 (2019) 172 [arXiv: 1901.02898] [INSPIRE].

[74] J.M. Henn, T. Peraro, M. Stahlhofen and P. Wasser, Matter dependence of the four-loop cusp anomalous dimension, Phys. Rev. Lett. 122 (2019) 201602 [arXiv: 1901.03693] [INSPIRE].

[75] S. Moch, B. Ruijl, T. Ueda, J.A.M. Vermaseren and A. Vogt, On quartic colour factors in splitting functions and the gluon cusp anomalous dimension, Phys. Lett. B 782 (2018) 627 [arXiv: 1805.09638] [INSPIRE].

[76] T. Becher and M. Neubert, Infrared singularities of scattering amplitudes in perturbative QCD, Phys. Rev. Lett. 102 (2009) 162001 [Erratum ibid. 111 (2013) 199905] [arXiv: 0901.0722] [INSPIRE].

[77] D. Binosi, J. Collins, C. Kaufhold and L. Theussl, JaxoDraw: a graphical user interface for drawing Feynman diagrams. Version 2.0 release notes, Comput. Phys. Commun. 180 (2009) 1709 [arXiv: 0811.4113] [INSPIRE].

[78] G.P. Korchemsky and A.V. Radyushkin, Renormalization of the Wilson loops beyond the leading order, Nucl. Phys. B 283 (1987) 342 [INSPIRE].

[79] S. Moch, J.A.M. Vermaseren and A. Vogt, The three loop splitting functions in QCD: the nonsinglet case, Nucl. Phys. B 688 (2004) 101 [hep-ph/0403192] [InSPIRE].

[80] S. Moch, J.A.M. Vermaseren and A. Vogt, The quark form-factor at higher orders, JHEP 08 (2005) 049 [hep-ph/0507039] [INSPIRE].

[81] S. Moch, J.A.M. Vermaseren and A. Vogt, Three-loop results for quark and gluon form-factors, Phys. Lett. B 625 (2005) 245 [hep-ph/0508055] [INSPIRE].

[82] A. Vogt, S. Moch and J.A.M. Vermaseren, The three-loop splitting functions in QCD: the singlet case, Nucl. Phys. B 691 (2004) 129 [hep-ph/0404111] [INSPIRE].

[83] T. Becher, M. Neubert and G. Xu, Dynamical threshold enhancement and resummation in Drell-Yan production, JHEP 07 (2008) 030 [arXiv: 0710.0680] [INSPIRE].

[84] V. Ahrens, T. Becher, M. Neubert and L.L. Yang, Renormalization-group improved prediction for Higgs production at hadron colliders, Eur. Phys. J. C 62 (2009) 333 [arXiv: 0809.4283] [INSPIRE].

[85] V.A. Smirnov, Analytic tools for Feynman integrals, Springer Tracts Mod. Phys. 250 (2012) 1 [INSPIRE]. 